\title{
Sterben und Tod - Sind wesentliche Bereiche am Ende des Lebens nicht normiert oder undefinierbar?
}

\section{Problemstellung}

Anfang und Ende des menschlichen Daseins beschäftigen als zentrale und existenzielle Fragen die Menschen in sämtlichen Kulturen und durch alle Jahrhunderte. Geburt, Leben, Sterben und Tod bestimmen den Ablauf biologischen und nicht nur menschlichen Lebens. Das dem Menschen innewohnende Bewusstsein, die Möglichkeit zu vorausschauendem Denken und die Erkenntnis der eigenen Vergänglichkeit erzeugen den Wunsch, den Prozess des Sterbens und des Todes zu verstehen und bestimmen zu können. Während Aristoteles den Todeszeitpunkt durch den Verlust der vitalen Körperwärme zu definieren versuchte, ist nach japanisch-shintoistischer Auffassung der Mensch erst mit dem Absterben aller Organe tot ${ }^{1}$. Neben der reinen Feststellung des möglichst exakten Todeszeitpunkts stellt sich auf metaphysischer Ebene die Frage nach der Veränderung des individuellen Menschseins durch Sterben und Tod, sowohl in der persönlichen als auch der gesellschaftlich-kulturellen Auseinandersetzung mit diesem Themenkomplex. Vita mutatur, non tollitur (Das Leben wird nicht genommen, sondern verändert.) war eine Auffassung aus der Totenliturgie des frühen Mittelalters, einer Epoche, die sich intensiv mit Sterben und Tod auseinandergesetzt hat ${ }^{2}$. Eine konkrete Vorstellung, die das Gehirn als zentrales Organ zur Todesfeststellung in den Mittelpunkt der Betrachtung stellt, gab es im Mittelalter nicht ${ }^{3}$. Im christlichen Selbstverständnis der Neuzeit spielt die Vorstellung vom ewigen Leben durch den Tod eine zentrale Rolle ${ }^{4}$. Mit der alten Bezeichnung »exitus letalis« wurde verstanden, dass die Seele mit dem Tod des Menschen den Körper verlässt ${ }^{5}$. Ungeachtet der religiösen Vorstellungen von der Unsterblichkeit der menschlichen Seele enden das Menschsein und die aus diesem Menschsein abgeleitete Rechtsfähigkeit mit dem Tod. Die transzendentale, metaphysische Bedeutung des Lebens nach dem Tode im Mittelalter ist heutzutage durch die moderne Transplantationsmedizin für Zellen, Gewebe und Organe durch ein verändertes Weiterleben in einem anderen Organismus faktisch und real möglich geworden. Während also Organstrukturen des ursprünglichen Organismus in einem anderen Körper mit eigenem Menschsein und Bewusstsein weiterleben, ist das Menschsein des postmortalen Spenders »spätestens « nach der Explantation unwieder-

1 Vgl. Vultejus, Der Mensch ad cadaver, ZRP 1993, S. 435 (436).

2 Ohler, Sterben und Tod im Mittelalter, 2003, S. 49.

3 Frewer, Tagungsbericht - 30 Jahre Hirntod-Definition - Historische und ethische Aspekte, Ethik Med 1999, 114 (115).

4 Erklärung der Deutschen Bischofskonferenz und des Rates der Evangelischen Kirche in Deutschland zur Organtransplantation, 1990, S. 22: »Wer glaubt, ist bereits jetzt vom Tod zum Leben hinübergegangen (vgl. Joh 5,24; vgl. auch Röm 6,13).«.

5 Beck, Medizinrecht: Todeszeitpunkt und Organentnahme. Was nun? Gedanken zu Aspekten des neuen Transplantationsgesetzes vom 1.11.1997, Anaesthesist 1997, S. 988. 
bringlich verloschen. Damit verknüpft ist deshalb die Frage nach dem Tod, den Kriterien des Todes und der Feststellung des Todeszeitpunkts des ursprünglichen menschlichen Organismus, des so genannten Organspenders.

Die aktuellen Debatten um Transplantationen, Sterbehilfe und Patientenverfügungen zeigen auf, vor welchen Problemen der einzelne und die Gesellschaft bei der Wertung zu Fragen des Sterbens und des Todes stehen, da ein Spektrum unterschiedlichster philosophischer, ethischer, religiöser, theologischer, medizinischer, gesellschaftspolitischer und rechtlicher Auffassungen zu Sterben und Tod vertreten wird. Während im Mittelalter Sterben und Tod nach religiöser Überzeugung nicht als Ziel sondern als Durchgang betrachtet wurden und Sterben- bedingt durch Seuchen wie die Pest und andere zum damaligen Zeitpunkt nicht behandelbare Krankheiten, eine hohe Säuglingsund Kinder- sowie Kindbettsterblichkeit - allgegenwärtig war, ist in unserer Leistungsund Spaß-Gesellschaft überwiegend kein Raum für eine Sterbe- und Trauerkultur ${ }^{6}$. In unserer modernen Gesellschaft werden Sterben und Tod tabuisiert, Gedanken an die eigene Vergänglichkeit verdrängt. Daran vermögen auch Körperweltenausstellungen, die den Tod zum Event und den Toten zum Marketingprodukt machen, nichts zu ändern, denn die groteske Inszenierung der Vergegenständlichung des plastinierten Toten macht den realen Sterbeprozess und den Tod des Menschen wirklichkeitsfremd und nicht transparent. Gleichzeitig dominiert das Recht auf individuelle Selbstbestimmung die Suche nach Lösungen für den Beginn des eigenen Sterbens und die Frage nach dem Recht auf den eigenen Tod. Jaspers beschreibt das Dilemma des modernen Menschen als doppelte Angst vor dem Sterben, nämlich der Angst vor dem »Nichtmehr-existieren « und vor dem »Nicht-sterben-können «?

Patzelt weist auf die unscharfen Begrifflichkeiten vom Menschen und dem menschlichen Leben hin, die die Debatte um die Grenzbereiche des menschlichen Lebens am Lebensende bestimmen ${ }^{8}$. So regelt das Bürgerliche Gesetzbuch zwar in § 1 BGB den Beginn der Rechtsfähigkeit des Menschen aber trotz der Scheintodängste im 19. Jahrhundert nicht dessen Ende, da der Tod als Ende der Rechtsfähigkeit ohne nährere Definition wie selbstverständlich vorausgesetzt wurde ${ }^{9}$. Während der Todesbegriff lange Zeit als überwiegend medizinisch-naturwissenschaftlich determiniert betrachtet und einer eindeutigen rechtlichen Wertung entzogen wurde ${ }^{10}$, ist seit der Hirntodde-

6 Vgl. auch Payk, Töten aus Mitleid? Über das Recht und die Pflicht zu sterben, 2004, S. 11.

7 Karl Jaspers zitiert nach Uhlenbruck/Ulsenheimer, Die ärztliche Sterbehilfe, in Laufs/ Uhlenbruck (Hrsg.) Handbuch des Arztrechts, 3. Aufl. 2002, § 132 Rn. 1.

8 Patzelt, Grenzbereiche des menschlichen Lebens, Rechtsmedizin 2004, S. 247.

9 Aus den Entwürfen zum BGB wurde die ursprüngliche Ergänzung des $\S 1$ BGB »und endigt mit dem Tod « gestrichen. Vgl. Prot. VIII 106; BayObLG NJW-RR 1999, S. 1309 (1311): »Die Frage, wann der Tod eingetreten ist, hat der Gesetzgeber als naturwissenschaftlich feststehend und daher nicht als regelungsbedürftig angesehen.«; Soergel/Stein, BGB, 13. Aufl. 2002, § 1922 Rn. 3.

10 Friedrich Carl v. Savigny, System des heutigen Römischen Rechts, Bd. 2, 1840, 17: »Der Tod als die Gränze der natürlichen Rechtsfähigkeit ist ein so einfaches Naturereignis, daß derselbe nicht, wie die Geburt, eine genauere Feststellung seiner Elemente nöthig macht.«; vgl. Stellungnahme der Bundesregierung, BT-Drs. 11/7980, S. 29: »Es erscheint nach wie vor sinnvoll und angemessen, die Definition und die Kriterien zur Feststellung des Hirntodes Medizinern als Fachleuten zu überlassen.«. 
batte im Rahmen des Erlasses des Transplantationsgesetzes ein Streit um die Verfassungsmäßigkeit der Regelungen zum Todesbegriff und seiner Feststellung in rechtlicher Sicht entstanden ${ }^{11}$. Zudem wird von juristischer Seite zunehmend gefordert, dass Ärzten und anderen Naturwissenschaftlern kein Primat bei der Grenzziehung zwischen Leben und Tod zukommen soll ${ }^{12}$, sondern der Tod als Rechtsbegriff der Kompetenz des Rechtssystems und der normativen Konvention $\mathrm{zu}$ unterstehen hat ${ }^{13}$. Dagegen wird traditionell und aufgrund der medizinischen Kompetenz für die Bestimmung des maßgeblichen Zeitpunkt des Todes nach wie vor auf die Medizinwissenschaft verwiesen ${ }^{14}$. Eine vermittelnde Ansicht stellt auf das fördernde Zusammenwirken der Wissenschaftsdisziplinen $a b^{15}$.

Die Feststellung des Sterbebeginns und vom Ende der menschlichen Existenz ist eine zentrale Frage für die Diskussion um postmortale Transplantationen und einen Behandlungsabbruch am Lebensende. Noch Anfang des letzten Jahrhunderts war eine gesetzliche Definition des Todes überflüssig. Mit dem Stillstand des Herzens, des Kreislaufs und der Atmung war der Mensch in diesen Zeiten für jedermann erkennbar tot. Durch die Entwicklungen in der modernen Medizin ist dieser Zustand mit einer Rettung des Lebens überwindbar, so dass eine konkrete Festlegung, wann der Mensch nunmehr tot ist, erforderlich wurde. Intensivmedizin, Wiederbelebung, Herztransplantationen usw. haben dazu geführt, die Selbstverständlichkeit der Gleichsetzung von Herz- und Kreislaufstillstand mit dem Tod zu hinterfragen ${ }^{16}$. Die Möglichkeiten der modernen Medizin haben aus dem Tod als »unverrückbarem, fixierten Naturereignis, das es nur zu konstatieren galt ${ }^{17}$, einen variablen Sterbeprozess mit reversiblem oder irreversiblem Ausgang gemacht ${ }^{18}$. Nach allgemeiner Auf-

11 Höfling/Rixen in Höfling (Hrsg.) Transplantationsgesetz § 3 Rn. 9 f.; Klinge, Schlette, Das »widersprüchliche« Transplantationsgesetz, Jura 1997, S. 642 (643).

12 Anderheiden, »Leben« im Grundgesetz, KritV 2001, S. 353 (358).

13 Beckmann, Ist der hirntote Mensch eine »Leiche?«, ZRP 1996, S. 219 (220); Geilen, Medizinischer Fortschritt und juristischer Todesbegriff, in FS Heinitz 1972, S. 372 (396); Rixen, Todesbegriff, Lebensgrundrecht und Transplantationsgesetz, ZRP 1995, S. 461 (462); Steffen, Wieviele Tode stirbt der Mensch? NJW 1997, S. 1619 (1619); Christmann in Bamberger/Roth 2003, § 1922 Rn. 5; Deutsch, Medizinrecht 4. Aufl. 1999, Rn. 405: »Tod« ist ein rechtlicher Begriff, dessen Inhalt nicht einfach aus der Medizin übernommen werden kann.«.

14 Vgl. § 3 TPG; Brox, AT-BGB, 25. Aufl. 2001, Rn. 661; Hübner, BGB-AT, 2. Aufl. 1996, Rn. 127; Köhler, BGB-AT 2001, § 20 Rn. 4.

15 Von Münch, Staatsrecht II, 5. Aufl. 2002, Rn. 328.

16 Heun, Der Hirntod als Kriterium des Todes des Menschen - Verfassungsrechtliche Grundlagen und Konsequenzen, JZ 1996, S. 214 (214 f.).

17 Stein, a. a. O. Rn. 3 im weiteren aber unzutreffend, dass durch die bestehenden Re animationsmöglichkeiten auch bei »Toten das Gehirn in Gang gehalten « werden kann.

18 Medizinische Fallbeispiele: Ein Mann mittleren Lebensalters erleidet einen Herzinfarkt (Myokardinfarkt). Der Herzinfarkt ist bedingt durch einen Verschluss eines Herzkranzgefäßes (Verschluss bei Coronarstenose), das einen Großteil des Herzmuskels (Myokards) versorgt. Durch den Verschluss erfolgt eine Minderversorgung (Minderperfusion) des Herzmuskels mit Sauerstoff. Die reduzierte Tätigkeit des Herzmuskels führt zu einer fehlenden Blutversorgung im Kreislauf. Nach 1 Minute tritt Bewusstlosigkeit und kurz danach der Herz-Kreislauf-Stillstand ein. Wenn sich der Sachverhalt im Wald ereignet, in dem der Patient alleine spazieren geht, führen der Stillstand des Herz-Kreislauf-Systems und der 
fassung enden menschliches Leben ${ }^{19}$ sowie die Rechtsfähigkeit des Menschen mit dem $\operatorname{Tod}^{20}$. Umstritten ist hingegen, wie der Tod des Menschen zu definieren oder wann der Mensch tot ist ${ }^{21}$. Seit der Einführung des Transplantationsgesetzes im Jahre 1997 wird in Deutschland der Gesamthirntod zur Feststellung des Individualtodes als Zulässigkeitskriterium neben dem Herz-Kreislauf-Tod über mehr als 3 Stunden vor einer Organentnahme gewertet ${ }^{22}$.

Mit dem nachfolgenden Beitrag sollen die Begriffe »Sterben « und »Tod « aus medizinischer und rechtlicher Sicht erläutert werden.

\section{Definitionen des Sterbens und Todes in der Medizin}

Ein Fachbereich der Rechtsmedizin, die Thanatologie (griechisch: Thanatos = Tod), befasst sich u. a. mit den unterschiedlichen Definitionen des Todes (Scheintod, klinischer Tod, Teilhirntod, Hirntod, Individualtod, endgültiger Tod, Organtod, intermediäres Leben, totaler Tod, den Vorgängen des Sterbens und Nahtoderfahrungen), Fragen der Todesfeststellung (sichere und unsichere Zeichen des Todes) sowie physiologischen und pathophysiologischen Prozessen im menschlichen Organismus unmittelbar vor und nach dem Tod ${ }^{23}$. Bereits die medizinische Vielfalt der Begriffe macht deutlich, wie schwierig die Abgrenzung und das allgemeine Verständnis im Einzelfall sein können. In diesem Zusammenhang ist zwischen dem Tod als unterschiedlich definierter Zeitpunkt in dem individuell variierenden zeitlichen Ablauf des Sterbeprozesses und der sicheren Feststellung dieser unterschiedlich definierten Zeitpunkte, insbesondere der Feststellung der Irreversibilität (d. h. dass eine Reanimation aus

Ausfall der Atmung, also der klinische Tod, nach einigen wenigen Minuten zur Minderversorgung des Gehirns, was aufgrund der geringen Sauerstofftoleranz des Gehirns dessen Funktionsausfall, den Hirntod, bedingt. Wenn sich der Vorgang in einer Fußgängerzone ereignet, in der Passanten Erste Hilfe leisten und eine notärztliche Versorgung erfolgt, können je nach Ausmaß der Erkrankung und dem Zeitpunkt der Rettung ein Überleben mit oder ohne Defektzuständen oder ein Versterben resultieren. Der Tod des Patienten ist nach diesem Fallbeispiel somit von der Zufälligkeit der Lokalisation des Sterbebeginns abhängig. Bedingt durch den Ort, an dem sich der Herzinfarkt des Mannes ereignete, entfällt oder eröffnet sich der rettende Kausalverlauf und führt zur Reversibilität oder Irreversibilität des Sterbevorgangs.

19 Vgl. Murswiek in Sachs (Hrsg.) Grundgesetz, Art. 2 Rn. 142.

20 Vgl. Heinrichs in Palandt, BGB, 2004, 63. Aufl., § 1 Rn. 3.

21 Höfling, Um Leben und Tod: Transplantationsgesetzgebung und Grundrecht auf Leben, JZ 1995, S. 26 (30) sowie Beckmann a. a. O. S. 220 mit der Darstellung von vier Ebenen: 1. Subjekt des Todes, 2. Definition des Todes, 3. Kriterien des Todes, 4. Testverfahren zum Todesnachweis; Herrig, Die Gewebetransplantation nach dem Transplantationsgesetz, 2002, S. 73 mit Darstellung von drei Ebenen: 1. Todesbegriff als Definition des Todes im Rechtssystem, 2. Todeskriterien als Zeichen des definierten Todes, 3. Todesfeststellung zur Feststellung und zum Nachweis der Todeskriterien.

22 Vgl. § 3 Abs. 2 Nr. 2 TPG: Die Entnahme von Organen ist unzulässig, wenn nicht vor der Entnahme bei dem Organspender der endgültige, nicht behebbare Ausfall der Gesamtfunktion des Großhirns, des Kleinhirns und des Hirnstamms nach Verfahrensregeln, die dem Stand der Erkenntnisse der medizinischen Wissenschaft entsprechen, festgestellt ist.

23 Mattern, Rechtsmedizin, in Ökologisches Stoffgebiet, 3. Aufl. 1999, S. 307. 
medizinischer Sicht den Sterbeverlauf nicht mehr umkehren kann), zu differenzieren. Mitunter hängt, wie die aktuelle Debatte um den Hirntod aufzeigt, die rechtliche und medizinische Bewertung des Todes vom ideologischen und/oder pragmatischen Standpunkt ab.

\section{Beginn des Sterbevorgangs (Sterbeprozess)}

Mindestens genauso schwierig wie die Bestimmung des Todes mit dem Dissens und der Festlegung in der Phase des dissoziierten Hirntodes ist der Beginn des Sterbeprozesses medizinisch und rechtlich $\mathrm{zu}$ fassen. Von einer rein zellulären Basis aus betrachtet, stirbt der Mensch bedingt durch die beginnende Zellalterung bereits von Beginn seiner Geburt. Von dieser extensiven Beurteilung des Sterbeprozesses losgelöst, kann der Beginn des Sterbens mit derjenigen Erkrankung gleichgesetzt werden, die direkt oder indirekt den späteren Tod bedingt ${ }^{24}$. Die Problematik an dieser Definition ist jedoch, dass ausgehend von einer retrospektiven Betrachtung eine solche Bewertung tauglich und möglich ist. Für eine Bewertung des Sterbevorgangs zum exante Zeitpunkt erweist sie sich jedoch wieder als möglicherweise richtige oder falsche Prognosewertung mit einer mehr oder weniger größeren Wahrscheinlichkeit des falsch oder richtig. Eine Phase im Sterbeprozess, der dem Tod im Sekunden bis Stundenbereich vorausgeht, bezeichnet man als Agonie (= Todeskampf ${ }^{25}$ ). Die zeitliche Dauer der Agonie hängt von den Todesumständen des Einzelfalls ab. Der Sterbevorgang und die Agonie als solche sind unabhängig davon, wie lange sie dauern oder den einzelnen belasten, dem Leben zuzurechnen. In der Sterbephase der Agonie kann es zu den so genannten Nahtodeserfahrungen kommen, deren Auftreten vitales Gehirngewebe voraussetzt und u. a. auf Sauerstoffmangel des Gehirns, Endorphinausschüttungen usw. zurückgeführt wird $^{26}$. Auf den zunächst durch multifaktorielle Bedingungen (z. B. genetische Disposition des Menschen, negative und positive Auswirkungen von

24 Spittler, Gehirn, Tod und Menschenbild, 2003, S. 75.

25 Vgl. Madea/Dettmeyer/Schmidt, Thanatologie, in Madea (Hrsg.) Praxis der Rechtsmedizin 2003 S. 20 f.: Fehlende bzw. sehr kurze Agonie bei vollständiger oder überwiegender Zerstörung des menschlichen Körpers, kurze Agonie häufig bei gewaltsamen nicht natürlichen Todesfällen (z. B. Ertrinken, Erhängen, Erdrosseln, Erwürgen) oder bei natürlichen Todesfällen mit foudrayantem Verlauf (akuter Herzinfarkt, Hirnmassenblutung) und lange Agonie (z. B. chronische Krebserkrankungen im Endstadium). Die Bezeichnung Todeskampf, als schmerzhafter und quälender Prozess, ist insoweit etwas missverständlich. Vgl. Mueller, Der Tod und seine Feststellung in Mueller (Hrsg.) Gerichtliche Medizin, 2. Aufl. 1975: »Im ganzen ist das Wort »Todeskampf« vom Gesichtspunkt des Sterbenden aus nicht der richtige Ausdruck; nur in Ausnahmefällen wird ein regelrechter Todeskampf beobachtet, der bis zum Ableben dauert, z. B. bei schmerzhaftem Herzinfarkt.« Auch andere Erkrankungen, die mit Vernichtungsschmerzen einhergehen, oder nicht natürliche gewaltsame Tode können sich in einem schmerzhaften Todeskampf äußern, während der überwiegende Teil der Sterbevorgänge mit einer mangelnden Sauerstoffversorgung des Gehirns und einer Bewusstlosigkeit einhergehen, so dass der Tod nicht mehr bewusst erlebt wird. Prien/Hönemann, Der Tod und seine Feststellung in Brinkmann/Madea: Handbuch der gerichtlichen Medizin, Bd. 1, 2004, S. 4 mit vier Agonietypen zwischen Wiederherstellung des Lebens (Restitutio ad integrum) bis zum Individualtod und dem Hinweis, dass die Bezeichnung Agonie durch die mögliche Reversibilität erst postmortal erfolgen kann. 
Umweltfaktoren auf die gesundheitliche Disposition) vorbestimmten Beginn des Sterbeprozesses kann in verschiedenen Formen unmittelbar Einfluss genommen werden: Vorverlagerung (Suizid, ärztliche Fehlbehandlung, Behandlungsabbruch, Sterbehilfe), keine zeitliche Verschiebung (natürlicher Verlauf), Rückverlagerung des individuell genetisch bestimmten Sterbeprozesses (Reanimation, ärztliche Behandlung). Persönliche, ärztliche und sonstige Einflüsse im Sterbeprozess können wieder neue Verschiebungen bedingen, die erneut diese drei Möglichkeiten zur Veränderung des Sterbebeginns eröffnen.

\section{Herztod, Herz-Kreislauf-Tod oder klinischer Tod}

Herztod, Herz-Kreislauf-Tod oder klinischer Tod beschreiben den Stillstand des HerzKreislaufsystems und der Atmung, einen gegebenenfalls reversiblen Zustand, der bei entsprechender Wiederbelebung zur vollständigen und folgenlosen Wiederherstellung des Patienten führen kann. Im Vergleich mit dem Gesamthirntod, der irreversibel ist, kann der klinische Tod durch therapeutische Maßnahmen überwunden werden. Bei frustraner Reanimation oder natürlichem Verlauf allerdings geht der Herztod oder klinische Tod entsprechend der kurzen Sauerstofftoleranz des Gehirns nach wenigen Minuten in den Hirn- und Individualtod des Menschen über. Umgekehrt kann bei entsprechenden zerebralen Erkrankungsbildern zunächst der Hirntod eintreten, der nach einigen Minuten in den klinischen Tod durch Stillstand der Herzfunktion und Atmung übergeht. Während der Herztod früher mit dem Tod des Menschen gleichgesetzt wurde, stellt dieser durch die bereits angesprochenen Möglichkeiten in der Medizin zur Reanimation zunächst auch nur ein unsicheres Zeichen des Todes dar ${ }^{27}$. Teilweise wird ein Herzstillstand von 10 Minuten bei normaler Körpertemperatur aber als Äquivalent zum Hirntod bezeichnet und die Organentnahme bei dem »Non heart-beating donor « befürwortet. Eine solche Definition des Todes wird in Deutschland als mögliches, aber unsicheres Todeszeichen abgelehnt ${ }^{28}$.

\section{Teilhirntod, Gesamthirntod}

Vor der medizinischen Konkretisierung der Hirntodbegriffe ist klarstellend zu erläutern, dass diese Begriffe in der überwiegenden Zahl der Todesfälle keine Rolle spielen, sondern fast ausschließlich bei intensivmedizinischen Behandlungskonstellationen mit Fragen des Behandlungsabbruchs und/oder von Organtransplantationen relevant werden. Die überwiegende Anzahl der ca. 830.000 Todesfälle pro Jahr ${ }^{29}$ und

26 Linke, Das Gehirn, 1999, S. 10 ff.; Prien/Hönemann a. a. O. S. 11; Spittler, Die Diskussion um den Hirntod - ein Perpetuum mobile? Ethik Med 1998, S. 60 (61), Spittler a. a. O. S. 68 ff.

27 Angstwurm, Todesdefinition sowie Hinweise zum Hirntod, Teilhirntod und Herztod, Internist 1999, S. 283 (284); Bajanowski/Brinkmann, Todesbegriffe und Todesfeststellung, in Brinkmann/Madea (Hrsg.) Handbuch der gerichtlichen Medizin, Bd. 1, 2004, S. 14; Madea/Dettmeyer/Schmidt a. a. O. S. 22.; Mattern a. a. O. S. 307.

28 Vilmar/Brandt/Hanrath/Haverich, Organentnahme nach Herzstillstand («Non heart-beating donor «), Dt. Ärztebl. 1998, A-3235.

29 Statistisches Bundesamt, Statistisches Jahrbuch 2003, Gestorbene 2001, S. 73. 
deren Zeitpunkte werden ärztlich nach den klassischen Todeskriterien (sichere Zeichen des Todes ohne Hirntodkriterien) und den Todesumständen diagnostiziert ${ }^{30}$. Die Zahl der Organspender, denen postmortal Organe entnommen wurden, bewegt sich pro Jahr dagegen insgesamt nur bei ca. 1000 bis 1200 in den Jahren 1995 bis $2003^{31}$.

Von entscheidender Bedeutung für die Bewertung des Hirntodes ist die zentrale Funktion des Gehirns für die Steuerung des gesamten menschlichen Organismus. Obwohl das Gehirn nur etwa $2-3 \%$ des Körpergewichts ausmacht, beansprucht es etwa $20 \%$ des gesamten Grundumsatzes. Während das Herz innerhalb mehrerer Minuten reanimierbar ist, lässt die geringere Sauerstofftoleranz des Gehirns nur eine Wiederbelebung innerhalb weniger Minuten $\mathrm{zu}^{32}$. Als hochkomplexes Organ dient das menschliche Gehirn u. a. der Wahrnehmung von Sinnesinformationen, dem emotionalen menschlichen Erleben und Bewusstsein, kommunikativen menschlichen Fähigkeiten sowie allen Denkfunktionen und Handlungsimpulsen ${ }^{33}$.

Bereits Bichat hat 1796 in seiner physiologischen Forschungsschrift Zusammenhänge zwischen der Unterbrechung der Hirntätigkeit (la mort du système nerveux) und der zeitlichen Abhängigkeit der Funktionsausfälle von Blutkreislauf, Atmung und Hirntätigkeit dargelegt ${ }^{34}$. 1959 wurde in Frankreich erstmals ein Zustand bei einem Patienten beschrieben, dessen Kreislauf und Beatmung künstlich aufrecht erhalten wurden, ohne dass Hirnfunktionen klinisch festzustellen waren ${ }^{35} .1968$ befasste sich eine Kommission der Harvard-Universität mit ersten formalen Kriterien zur Feststellung des Hirntodes, der von der Kommission als »irreversible coma« (nicht rückbildungsfähiges Koma) bezeichnet wurde, um primär der Bedeutung des Krankheitsbildes für den zu behandelnden hirntoten Patienten und den möglichen Behandlungsabbruch, sekundär auch den Interessen der sich stetig entwickelnden Transplantationsmedizin Rechnung zu tragen ${ }^{36}$. In den Entscheidungshilfen der Bundesärztekammer wurde der Hirntod zunächst unmit-

30 Madea/Henssge/Dettmeyer, Hirntod als allgemeiner Todesbegriff, MedR 1999, S. 162.

31 Deutsche Stiftung Organtransplantation, Organspende und Transplantation in Deutschland, 2004 S. 8: insgesamt 1140 tote Spender in 2003.

32 Spittler a. a. O. S. 55

33 Spittler a. a. O. S. $29 \mathrm{ff}$.

34 Bichat, Recherches physiologiques sur la vie et la mort. 1796. Frewer a. a. O. S. 115: Nach Ausführungen von Métraux hat Bichat aber keine Aussagen zum Tod des Gehirns als Tod des Menschen getroffen, da er den Begriff »a mort du système nerveux « anstatt »mort cérébrale« gewählt hat ebenso Schlich, Ethik und Geschichte: Die Hirntoddebatte als Streit um die Vergangenheit, Ethik Med 1999, S. 79 (82).

35 Mollaret/Goulon, Le coma dépassé, Rev. Neurol. 1959, S. 3 ff. : Nach dem Verständnis der damaligen Autoren stellte aber erst der Herztod den Ende des Lebens des Patienten dar.

36 Beecher et al., A definition of irreversible coma - report of the ad hoc committee of Harvard medical school to examine the definition of brain death, JAMA 1968, S. 337 ff.; a. A. Jonas, Gehirntod und menschliche Organbank: Zur pragmatischen Umdefinierung des Todes, in Jonas (Hrsg.) Technik, Medizin und Ethik. 1987, S. 219 ff., der ursprünglich der Harvard-Kommission angehört hat und diese wegen dem Übergewicht der chirurgischen Interessen verlassen haben soll. Trotz der Zunahme der Spendebereitschaft besteht für Deutschland eine deutliche Diskrepanz zwischen Nierentransplantationen $(\mathrm{n}=2516)$ und Patientinnen und Patienten auf der Warteliste $(\mathrm{n}=9479)$ siehe Organspende und Transplantation in Deutschland 2003, Deutsche Stiftung Organtransplantation 2004. 
telbar mit dem Tod des Menschen gleichgesetzt ${ }^{37}$, in späteren Darstellungen dann aber auf den naturwissenschaftlich-medizinischen Feststellungszusammenhang reduziert ${ }^{38}$.

Zur Zeit werden verschiedene Hirntodkonzepte vertreten, die medizinisch und rechtlich zur Feststellung des Individualtodes umstritten sind. In Großbritannien wird das Hirnstamm-Todeskonzept (»brainstem-death «) angewandt, das dem Ausfall aller Hirnstammreflexe Rechnung trägt, ohne der Großhirnfunktion weitergehende Beachtung zu schenken. Bei diesem Konzept wird davon ausgegangen, dass dem Großhirn durch die totale Schädigung des Hirnstamms keine seelisch-geistige Funktion mehr zukommt $^{39}$. Haupt stellt klar, dass diese englischen Kriterien im internationalen Vergleich von Hirntodrichtlinien als Sonderweg zu betrachten sind, der sich aus pragmatischen Erwägungen und organisatorischen Gegebenheiten des englischen Medizinsystems ergeben hat ${ }^{40}$.

Als anthropologisches Konzept wird der Großhirntod (»neocortical death «) oder Kortikaltod vertreten. Es stellt auf den Ausfall aller Großhirnfunktionen und somit auf den Verlust des Großhirns als Sitz der seelisch-geistigen Aktivität $\mathrm{ab}^{41}$. Bei anenzephalen Neugeborenen liegen unterschiedlich ausgeprägte Missbildungen des Gehirns (bis zum völligen Fehlen des Großhirns) bei funktionstüchtigem Hirnstamm und Atmungs- sowie Herzkreislauffunktion vor. Diese Neugeborenen haben nur eine geringe Überlebenszeit von Stunden bis Wochen. Aus medizinischer Sicht wird insbesondere wegen der möglichen Organspende teilweise vertreten, diese Neugeborenen trotz des intakten Hirnstamms Hirntoten gleichzustellen ${ }^{42}$. Tendenzen in den USA deuten auf eine zunehmende Favorisierung eines Teilhirnkonzepts in der medizini-

37 Wissenschaftlicher Beirat der Bundesärztekammer, Kriterien des Hirntodes - Entscheidungshilfen zur Feststellung des Hirntodes. Dt Ärztebl. 1982, S. 35 ff.; 1986, S. B. 2940 ff.; 1991, S. B. 2855 ff.: »Der Hirntod ist der Tod des Menschen«.

38 Wissenschaftlicher Beirat der Bundesärztekammer, Richtlinien zur Feststellung des Hirntods. Dritte Fortschreibung 1997 mit Ergänzungen gemäß Transplantationsgesetz (TPG). Dt Ärztebl 1998 A 1861 ff.: »Mit dem Hirntod ist naturwissenschaftlich-medizinisch der Tod des Menschen festgestellt«.

39 Conference of royal colleges and faculties of the United Kingdom, Diagnosis of brain death, Lancet 1976, S. 1069 f. Nach Spittler a. a. O. S. 77 ff. können bei diesem funktionell und strukturell gut definierten Konzept medizintheoretisch Abgrenzungsschwierigkeiten beim totalen Locked-In-Syndrom auftreten. Beim Locked-In-Syndrom kann infolge einer Blutung oder Durchblutungsstörung im Hirnstammbereich bei erhaltener Bewusstseinslage der Verlust sämtlicher Bewegungsfunktionen des Körpers bedingt sein. Der Patient ist trotz des bedauerlichen Zustands bei Bewusstsein und als lebendig anzusehen, so dass eine Beendigung der Beatmung als Euthanasie zu werten wäre.

40 Haupt, Hirntodkonzeption und Hirntoddiagnostik aus neurologischer Perspektive, in Höfling (Hrsg.), Transplantationsgesetz, 2003, Anhang 1 zu § 16 Rn. 33.

41 Darstellung des theoretischen Konzepts bei Spittler a. a. O. S. 80, der die funktionell und strukturell fehlende Trennschärfe und schwierige Diagnostizierbarkeit aufzeigt. Abgrenzungsprobleme können sich beim apallischen Syndrom und bei anenzephalen Neugeborenen zeigen; Kurthen/Linke/Reuter, Hirntod, Großhirntod oder personaler Tod? Zur aktuellen Diskussion um die hirnorientierte Todesbestimmung. Med Klin 1989, S. 483 ff.

42 Holzgreve/Beller et al., Kidney transplantation from anencephalic donors, New Eng J Med 1987, S. 1069 f. siehe auch weitere medizinische Quellen bei Kloth, Anenzephale als Organspender - Eine rechtsvergleichende Betrachtung, MedR 1994, S. 180 Fn. 6. 
schen Wissenschaft hin ${ }^{43}$. Unter Darstellung des Wachkomas von Apallikern und der sozialen Reintegrierung bei entsprechender ärztlicher, pflegerischer und stimulativer Betreuung wird ein direkter Vergleich von Apallikern mit Hirntoten oder Teilhirntoten abgelehnt ${ }^{44}$.

Der Gesamthirntod wird als irreversibler Verlust aller Hirnfunktionen definiert, während Teilhirntod lediglich den irreversiblen Verlust des Großhirns oder des Hirnstamms beschreibt. Beim Gesamthirntod sind alle Funktionen des Großhirns, des Kleinhirns und des Hirnstamms irreversibel erloschen. Trotz des Gesamthirntodes ist es mitunter möglich, intensivmedizinisch Herz, Kreislauf und Atmung apparativ und medikamentös noch aufrecht zu erhalten (dissoziierter Hirntod) ${ }^{45}$.

Die Feststellung des Gesamthirntodes bedeutet also einen irreversiblen Funktionsausfall der Gesamtfunktion des Großhirns, Kleinhirns und Hirnstamms, ohne dass eine vollständige Gewebsnekrose, ein vollständiger Zirkulationsabbruch, ein restloser und vollständiger Funktionsverlust von Zellen und Zellverbänden im Gehirn oder im Bereich des Zwischenhirns, der Hypophyse oder des Hypothalamus vorliegen muss ${ }^{46}$.

Rechtsmedizinische Studien belegen, dass auf zellulärer Ebene ein Absterben der Nervenzellen erst nach bis zu 72 Stunden erfolgen $\mathrm{kann}^{47}$, wobei in Abhängigkeit von verschiedenen Faktoren und der zugrundeliegenden Erkrankung ein schneller und langsamer Zelltod beschrieben wird ${ }^{48}$. Dem klinischen Bild des Hirntodes im Minutenbereich kann sich eine zelluläre Reaktion von einigen Tagen anschließen ${ }^{49}$, so dass bei einer Obduktion unmittelbar nach dem Hirntod ein morphologisches Korrelat für den Hirntod nicht zwangsläufig zu finden ist.

Die Feststellung des Hirntodes wird von Schwerdt als schwerwiegendste ärztliche Diagnose und als Todesurteil beschrieben ${ }^{50}$. Von rechtsmedizinischer Seite wird der

43 Vgl. in der Schmitten, Organtransplantation ohne »Hirntod«-Konzept? Ethik Med 2002, S. $60(61)$.

44 Dörner, Hält der BGH die »Freigabe der Vernichtung lebensunwerten Lebens « wieder für diskutabel?, ZRP 1996, S. 93 ff.

45 Spittler a. a. O. S. 33.

46 Spittler a. a. O. S. 8; Wissenschaftlicher Beirat der Bundesärztekammer, 1998 a. a. O. A1865: Kein zerebraler Zirkulationsstillstand z. B. bei großen offenen Schädel-Hirn-Verletzungen oder bei sekundären Hirnschädigungen.

47 Tomimoto/Yanagihara, Vulnerability of dendrites and nerve cell bodies in cerebral ischemia, in Oehmichen (Hrsg.) Brain Hypoxia and Ischemia, Research in Legal Medicine, Vol 24, 2000, S. $65 \mathrm{ff}$.

48 Smith, Injury-determing factors and cellular mechanisms for ischemic damage in the brain, in Oehmichen (Hrsg.) Brain Hypoxia and Ischemia, Research in Legal Medicine, Vol 24, 2000, S. $59 \mathrm{ff}$.

49 Oehmichen/Meissner, Forensic neuropathological aspects of cerebral anoxia/ischemia and hypoxia/hypoxemia, in Oehmichen (Hrsg.) Brain Hypoxia and Ischemia, Research in Legal Medicine, Vol 24, 2000, S. $13 \mathrm{ff}$.

50 Schwerdt, Rechtsmedizin, 5. Aufl. 1992, S. 183. Hierbei ist jedoch zu berücksichtigen, dass ein Todesurteil nur gegen einen Lebenden verhängt werden kann. Somit könnte der Eindruck entstehen, dass ein Lebender aufgrund der Einschätzung des Arztes getötet wird. Richtiger wäre im Gesamtkontext der Ausführungen von Schwerdt deshalb davon zu sprechen, dass die ärztliche Diagnose des Hirntods die Feststellung des Todes nicht aber das Todesurteil bedeutet. 
Gesamthirntod überwiegend mit dem Individualtod und dem individuellen Aktivitätszustand tot gleichgesetz $\mathrm{t}^{51}$. In einer gemeinsamen Erklärung verschiedener Vorsitzender medizinischer Gremien und Gesellschaften werden die biologisch begründete Definition des Hirntodes, die Sicherheit der Hirntodfeststellung und die Bedeutung des Hirntodes als sicheres inneres Todeszeichen unterstrichen ${ }^{52}$. Förderreuther und Angstwurm bezeichnen die Feststellung des Hirntodes als Dokumentation eines sicheren inneren Todeszeichens ${ }^{53}$. Patzelt beschreibt den Körper des Hirntoten als menschliche Leiche mit noch erhaltener Supravitalität und postmortaler Würde und definiert die Zeitspanne zwischen Hirntod und biologischem Tod als »menschliches Leben ohne Qualität zum Menschsein $\aleph^{54}$. Der endgültige Ausfall der gesamten Hirnfunktion wird in der herrschenden Auffassung der Medizin daher als sicheres Todeszeichen und Individualtod des Menschen gewertet ${ }^{55}$.

Beck vertritt hingegen, dass der hirntote Patient nicht mit einer Leiche gleichgesetzt werden kann, sich aber irreversibel im Vorfeld des Todes befindet. Er beschreibt wei-

51 Bajanowski/Brinkmann a. a. O. S. 17: »Der Hirntod wird dem Individualtod gleichgesetzt, der aus rechtlicher Sicht den Zeitpunkt darstellt, zu dem das Leben eines Menschen als »bewusstseins- und selbstbewusstseinsfähiges Lebewesen « beendet ist. (Interessanterweise heben die Autoren die rechtliche und weniger die medizinische Sichtweise bei dieser Definition des Hirntodes hervor); Forster/Ropohl, Thanatologie, in Forster (Hrsg.) Praxis der Rechtsmedizin für Mediziner und Juristen, 1986, S. 8: »Hirntod (= Individualtod)《 allerdings mit einer Schwebezeit zwischen beginnendem und vollendeten Hirntod mit der Differenzierung unsicherer und infauster Prognose; Madea/Henssge/Dettmeyer, a. a. O. S. 162 f; Schneider, Leichenschau, Rechtsmedizin 2002, 339 (341): »Der Individualtod ist dem Hirntod gleichzusetzen (Totalnekrose des Gehirns)«; Schwerdt, a. a. O. »Der Hirntod wird dem Individualtod gleichgesetzt $\ll$.

52 Gemeinsame Erklärung zum Hirntod des Präsidenten der Deutschen Gesellschaft für Anaesthesiologie und Intensivmedizin Götz, des 1. Vorsitzenden der Deutschen Gesellschaft für Neurochirurgie Oppel, des 1. Vorsitzenden der Deutschen Gesellschaft für Neurologie Hacke, des Präsidenten der Bundesärztekammer und des Deutschen Ärztetages Hoppe und des Vorsitzenden des Wissenschaftlichen Beirats der Bundesärztekammer Sewing, Dt. Ärztebl. 2001, A 1417: »Übereinstimmend auch mit der neueren wissenschaftlichen Literatur wird gegenüber anders lautenden und missverständlichen Äußerungen leider auch einzelner Ärzte - klargestellt: 1. An der biologischen Definition des Hirntodes, an der Sicherheit der Hirntodfeststellung und an der Bedeutung des Hirntodes als sicheres inneres Todeszeichen des Menschen hat sich nichts geändert. 2. Nach dem Hirntod gibt es keine Schmerzempfindung mehr. Deshalb sind nach dem Hirntod bei Organentnahmen keine Maßnahmen zur Schmerzverhütung (zum Beispiel Narkose) nötig...« a. A. Schlemmer, Dt. Ärztebl. 2001, A-1876; Dörner, Dt. Ärztebl. 2001, A-1877; ablehnende Erwiderung Lang, Dt. Ärztebl. 2001, A-2097; ablehnende Erwiderung auf Lang: Lotz, Dt. Ärztebl. 2001, A-2640; Ritz, Dt. Ärztebl. 2001, A-2640 mit einer völlig fehlerhaften Darstellung des Hirntodes: »Beim Hirntod ist in der Regel nur ein kleiner, wenn auch wichtiger Teil des Gehirns dauerhaft ausgefallen, die Hirntoten sind aber durchaus in der Lage, ein »subkortikales« Leben zu führen; klarstellende Kritik zur fehlerhaften Darstellung von Ritz durch Frank, Dt. Ärztebl. 2001, A-2872; Birk, Dt. Ärztebl. 2001, A-2874 und Wöhrle/ Quintel, Dt. Ärztebl. 2002, A-350; Klarstellende Äußerungen durch Hacke/Schildberg/ Oppel, Dt. Ärztebl. 2002, A-624.

53 Förderreuther/Angstwurm, Anaesthesie zur Organentnahme. Anaesthesist 2002, S. 673; Angstwurm, a. a. O. S. 284.

54 Patzelt a. a. O. S. 249. 
terhin den Hirntoten als Sterbenden, der den vollen Schutz der Menschenwürde und körperlichen Unversehrtheit verdien ${ }^{56}$. Bavastro führt aus, dass der hirntote Mensch schwerstkranker Sterbender aber nicht bereits Toter sei und Leben noch vorhanden nur aber wach-rationales gegenständliches Bewusstsein erloschen sei ${ }^{57}$. Unger schlägt vor, die Begrifflichkeit des Hirntodes durch irreversibles Hirnversagen (»Coma egressum«) zu ersetzen ${ }^{58}$. Hamann und Gärtner warnen, dass klinisch ein tiefes Koma mit Ausfall der Hirnstammreflexe und vegetativer Entgleisung zunächst nicht von einem Hirntodsyndrom unterschieden werden kann $^{59}$. Kretz und Schäffer hingegen konstatieren, dass »allein schon durch neurologische Untersuchungen der Hirntod irrtumsfrei feststellbar ist ${ }^{60}$. Aus intensivmedizinisch-pflegerischer Sicht wird auf das Dilemma hingewiesen, dass die Versorgung von Toten keine pflegerische Aufgabe ist ${ }^{61}$. Aus intensivmedizinisch-ärztlicher Sicht wird die Rechtssicherheit der Organentnahme bei Hirntoten unterstrichen, aber auf ethische Probleme der veränderten Arzt-PatientenBeziehung mit Aufgabenverlagerung durch die Drittnützigkeit der Organspende für den Organempfänger hingewiesen ${ }^{62}$. Im Hinblick auf die Feststellung des Hirntodes und die Optimierung eines postmortalen potentiellen Spenders vor der Organentnahme sind die Ausführungen von Klöss et al. kritisch zu werten, die zudem das schrittweise Erlöschen der zerebralen Funktion anführen ${ }^{63}$. Gruber kritisiert die zunehmende Pra-

55 Vgl. Wissenschaftlicher Beirat der Bundesärztekammer, a. a. O.; Lang, Kultur des Lebens oder Kultur des Sterbens, ZRP 1995, S. 457 (459); Spittler, Der menschliche Körper im Hirntod, ein dritter Zustand zwischen lebendem Menschen und Leichnam, JZ 1997 S. 747 (751).

56 Beck a. a. O. S. 990.

57 Bavastro, Das Hirnversagen und das Transplantationsgesetz, ZRP 1999, S. 114 (116 f.).

58 Unger, Das Coma egressum - der irreversible Hirnausfall, MMW 1995, S. 510.

59 Hamann/Gärtner, Komata aus internistischer und neurologischer Sicht, Internist 2001, S. 956 (959).

60 Kretz/Schäffer, Anästhesie, Intensivmedizin, Notfallmedizin, Schmerztherapie, 2000, 3. Aufl. S. 517: In den weiteren Ausführungen sprechen sie dann davon, dass »der Hirntod letztendlich als Tod des Hirnstamms definiert wird «, was eher dem brainstem-Konzept nach englischem Verständnis als dem Ganzhirntod nach deutschem Muster trotz des Verweises auf die Richtlinien der Bundesärztekammer entspricht.

61 Holthaus, Die Pflege von Hirntoten aus der Sicht eines Intensivpflegers, Ethik der Medizin 2000, S. 247 (255): »Sehen wir den Hirntoten als lebenden Menschen an, sind Pflegeziele zu erkennen, die eine pflegerische Intervention nötig machen. (...) Sehen wir den Hirntoten als Toten an, muss die Pflege zum Zeitpunkt der Hirntodfeststellung enden.«

62 Schuster, Ethische Probleme im Bereich der Intensivmedizin, Internist 1999, S. 260 (266) mit Befürwortung der Transplantation bei Hirntoten auf der Basis der individuellen Abwägung des einzelnen Arztes (»Jede Ärztin und jeder Arzt in der Intensivmedizin muss dies mit sich selbst ausmachen.«).

$63 \mathrm{Klöss} / \mathrm{Castan} /$ Simmendinger, Maßnahmen zur Konditionierung eines postmortalen potenziellen Spenders vor der Multiorganentnahme, Intensivmedizin 2001, S. 64 (64, 65, 69): »Im Allgemeinen beginnen wir die Intensivbehandlung potentieller Organspender nach der klinischen Dezerebration, die zusätzlich durch eine Dopplersonographie der intrakraniellen Arterien erhärtet wird. Diese Untersuchung kann zwar einmalig den Hirntod nicht beweisen, gibt aber zusammen mit den klinischen Zeichen gute Hinweise, auf das Erlöschen der intrakraniellen Zirkulation... Bis zur endgültigen Feststellung des Hirntods werden diese Patienten gepflegt und versorgt. Auch während der Wartezeit bis zur operativen Entnahme (...) muss die oben beschriebene Diagnostik und Stabilisierungstherapie konsequent weiterlaufen.« Auf 
xis der ambulanten Hirntoddiagnostik durch mobile Untersuchungsteams ${ }^{64}$ zur Verkürzung der Beobachtungszeit der Hirntoddiagnostik und zur primären Rekrutierung potentieller Organspender und spricht sich für eine strikte Trennung der Verantwortungsbereiche Hirntoddiagnostik und Organgewinnung aus ${ }^{65}$.

Als Todeszeitpunkt gelten bei der Hirntoddiagnostik in Deutschland und der Schweiz der Abschluss der Diagnose und Dokumentation des Hirntodes, in Österreich hingegen der Eintritt des Herzkreislaufstillstandes des hirntoten Organspenders ${ }^{66}$.

\section{Totaler oder biologischer Tod}

Dem totalen oder biologischen Tod geht eine Zeitspanne voraus, die als intermediäres Leben bezeichnet wird und dem Individualtod des Menschen folgt. In der Zeitspanne des intermediären Lebens lassen sich körperliche Reaktionen, so genannte supravitale Reaktionen, wie Muskelkontraktionen oder pharmakologische Effekte, z. B. Pupillenreaktionen nach Adrenalingabe, als Reaktion von Zellen und Zellsystemen auslösen. Zentrale Regulationsmechanismen durch das Gehirn oder das Herz-Kreislaufsystem bestehen während der Phase des intermediären Lebens aber unumkehrbar nicht mehr ${ }^{67}$. In diesem Zeitraum erfolgt ein weiteres Absterben von Organsystemen. Der Begriff Leben bezeichnet in diesem Zusammenhang somit nicht mehr das Leben eines Individuums sondern nur noch einzelner menschlicher Zellen oder Organstrukturen. Totaler oder biologischer Tod bezeichnet das Absterben der letzten Zelle eines menschlichen Organismus. Mit dem Absterben der letzten Zelle ist der Sterbeprozess $\mathrm{zu}$ Ende gebracht ${ }^{68}$. Aus praktischen gesellschaftlichen, medizinischen und rechtlichen Erwägungen (z. B. Todesfeststellung für Bestattung, Erbrecht, Transplantation) wird der totale oder biologische Tod als letztendlicher Todeszeitpunkt des menschlichen Lebens nicht herangezogen, sondern im Prozess des Sterbens ist ein zu definierender Zeitpunkt, ein point of no return, zu bestimmen, der einerseits den möglicherweise reversiblen Scheintod ausschließt und andererseits den irreversiblen Individualtod beschreibt ${ }^{69}$.

grund dieser wissenschaftlichen Darstellung stellt sich die Frage, ob nicht im besonderen die Organoptimierung auch vor den Zeichen der klinischen Dezerebration durchgeführt wird und inwieweit intensivmedizinisch und transplantationsmedizinisch sinnvoller Pragmatismus mit den strengen Anforderungen der unabhängigen Hirntodfeststellung vereinbar sind.

64 Vgl. auch Hinweise auf überregionale Konsiliardienste durch Anforderung über die regional zuständigen Organisationszentralen der Deutschen Stiftung Organtransplantation bei: Förderreuther/Angstwurm a. a. O. S. 675.

65 Gruber, Meldepflicht potentieller Organspender, ZRP 1998, S. 127 ff.

66 Sinner/Graf, Anaesthesie zur Organentnahme, Anaesthesist 2002, S. 498 ff.

67 Forster/Ropohl a. a. O. S. 11; Madea/Krause/Jachau a. a. O. S. 41; Mattern a. a. O. S. 310; Schwerdt a. a. O. S. 188.

68 Prien/Hönemann, a. a. O. S. 1; Schneider, a. a. O. S. 341.

69 Definition des wissenschaftlichen Beirats der Bundesärztekammer: »Der Organismus ist tot, wenn die Einzelfunktion seiner Organe und Systeme sowie ihre Wechselbeziehungen unwiderruflich nicht mehr zur übergeordneten Einheit des Lebewesens in seiner funktionellen Gesamtheit zusammengefasst und unwiderruflich nicht mehr von ihr gesteuert werden.« Dt. Ärztebl. 1993, 2933. 


\section{Feststellung des Todes bei der Leichenschau (unsichere und sichere äußere Zei- chen des Individualtodes) und Feststellung des Hirntodes}

Unsichere Zeichen des Todes (vita reducta, vita minima) hingegen, wie Blässe der Haut, fehlende Atmung, Herz-Kreislauf-Stillstand, fehlende Pupillenreaktionen und Reflexe können Anzeichen eines klinischen Todes oder Herz-Kreislauftods sein, der unter Berücksichtigung der Sauerstoffempfindlichkeit der verschiedenen Organe ${ }^{70}$ bei adäquater medizinischer Wiederbelebung reversibel sein kann. Gerade das Vorhandensein unsicherer Todeszeichen kann bei Intoxikationen, Traumata und verschiedenen Erkrankungen zur diagnostischen Fehleinschätzung eines Scheintods führen ${ }^{71}$. Beim Scheintod kann eine erfolgreiche Wiederbelebung abhängig von der zugrunde liegenden Ursache möglich sein.

Die sicheren äußeren Zeichen des Todes zeigen als Feststellungskriterien bei der Leichenschau auf, dass der Sterbeprozess des Patienten unumkehrbar und irreversibel tödlich verlaufen ist. Das Leben des menschlichen Individuums ist durch den Individualtod beendet worden, der unwiderruflich feststeht, wenn sichere Todeszeichen wie Totenflecken (Livores), Totenstarre (Rigor mortis) und/oder Fäulnis nachweisbar sind oder eine mit dem Leben nicht zu vereinbarende Zerstörung des Körpers durch Exenteration lebenswichtiger Organe stattgefunden hat ${ }^{72}$. Die äußerlich sichtbaren Totenflecken entstehen als das am frühesten auftretende sichere Todeszeichen durch die schwerkraftbedingte Hypostase des Blutes als Folge des irreversiblen Herz-KreislaufStillstandes. Die Variationsbreite des zeitlichen Beginns wird mit 15 Minuten bis zu 180 Minuten angegeben ${ }^{73}$. Die Totenstarre als weiteres sicheres Todeszeichen tritt im Mittel 3 bis 4 Stunden nach dem Tod als Folge eines Abfalls der ATP-Konzentration im Muskel und einer irreversiblen Verbindung zwischen Aktinfilamenten und Myosinköpfchen auf, was zu einer Versteifung der Muskulatur führt. Die Feststellung der sicheren Todeszeichen ist nicht mit der Feststellung des Todeszeitpunktes gleichzusetzen, denn die sicheren Zeichen des Todes treten erst mit einer zeitlichen Latenz nach dem Tod des Menschen auf. Im Rahmen der Leichenschau ist deshalb zwischen der Feststellung des Todes und der Ermittlung der Todeszeit zu differenzieren ${ }^{74}$.

70 Vgl. bereits Forster/Ropohl a. a. O. S. 11 mit der Darstellung experimenteller Wiederbelebungszeiten verschiedener Organe.

71 Bahrmann; Ardab-Zadeh; Mattern; Madea/Dettmeyer/Schmidt a. a. O. S. 21 mit Falldarstellung einer ärztlichen Fehlleistung bei der Todesfeststellung: Fall Minna Braun; Schäfer, Scheintod - Auf den Spuren alter Ängste, 1. Aufl. 1994 m. w. N.

72 Bajanowski/Brinkmann a. a. O. S. 14 ff.; Madea/Dettmeyer/Schmidt a. a. O. S. 20; Mattern a. a. O. $307 \mathrm{ff}$.; Schwerdt a. a. O. S. $184 \mathrm{ff}$.

73 Madea/Krause/Jachau in Madea (Hrsg.), Praxis Rechtsmedizin, 2003 S. 35 ff.

74 Während die Feststellung des Todes mit den sicheren Zeichen des Todes eindeutig erfolgen kann, stellt die Bestimmung des Todeszeitpunktes eine Schätzgröße dar, die um so ungenauer wird, je länger der tatsächliche Todeszeitpunkt zurückliegt. Temperaturabfall in der Leiche (Messung der Rektaltemperatur), Ausprägung und Wegdrückbarkeit der Totenflekken (Livores), zeitlicher Ablauf der Entwicklung der Totenstarre und Ausprägung der späten Leichenveränderungen wie Fäulnis und Autolyse dienen u. a. der Schätzung des maßgeblichen Todeszeitpunktes (vgl. Madea/Dettmeyer, Ärztliche Leichenschau und Todesbescheinigung, Dt. Ärztebl. 2003, A 3161 (3166); Schneider a. a. O. S. 342). 
Beim Vorliegen sicherer äußerer Todeszeichen ist der Sterbeprozess unumkehrbar in den Individualtod des Menschen gemündet, so dass eine Wiederbelebung nicht möglich ist. Deshalb wird bei der ärztlichen Leichenschau zur Feststellung des Todes verlangt, dass mindestens eines dieser Zeichen vorliegt.

Die Feststellung des Hirntodes erfolgt nach standardisierten Regeln unter Einschluss persönlicher ärztlicher Beobachtung und gegebenenfalls der Heranziehung spezieller insbesondere neurologischer apparativer Diagnostik ${ }^{75}$. Nach den Richtlinien zur Feststellung des Hirntodes des wissenschaftlichen Beirats der Bundesärztekammer ${ }^{76}$ erfor- $^{-}$ dert die Diagnose Hirntod die obligate Feststellung der Bewusstlosigkeit (Koma), der Hirnstamm-Areflexie und des Atemstillstandes (Apnoe) sowie den Nachweis der Irreversibilität der klinischen Symptome aufgrund entsprechend definierter Beobachtungszeiträume (Unterschiede in Abhängigkeit von Alter des Patienten und Form der Hirnschädigung (primär/sekundär)) oder durch geeignete ergänzende apparative Diagnostik (EEG zur Registrierung bioelektrischer Potentialschwankungen des Gehirns so genannte »Hirnströme«; evozierte Potentiale zur Registrierung von Potentialänderungen des Gehirns durch vorgegebene Reizung oder zerebrale Zirkulationskontrolle durch Dopplersonographie, zerebrale Perfusionsszintigraphie sowie Angiographie $)^{77}$.

\section{Definitionen des Sterbens und Todes in unterschiedlichen Rechtsgebieten}

Aus juristischer Sicht stellt sich die Frage, ob Begrifflichkeiten, wie Sterben oder Tod, einheitlich geregelt sind oder geregelt sein müssten. Schon der Beginn des Schutzguts »Leben« wird in den verschiedenen Rechtsgebieten unterschiedlich definiert und willkürlich aufgespaltet ${ }^{78}$. Verfassungsrechtliche Aspekte zum Würdeschutz des ungeborenen Lebens, zum Beginn der zivilrechtlichen Rechtsfähigkeit und des strafrechtlichen Lebensschutzes weisen zeitliche Unterschiede und verschiedene zugrunde liegende rechtliche Bewertungen auf. Während im BGB (§ 1 BGB) als Zeitpunkt der Rechtsfähigkeit des Menschen auf die Vollendung der Geburt, also den vollständigen Austritt aus dem Mutterleib abgestellt wird, sind strafrechtlich nach der positiv-rechtlichen Wertung des inzwischen aufgehobenen $\S 217$ StGB $^{79}$ für das

75 Wissenschaftlicher Beirat der Bundesärztekammer, Richtlinien zur Feststellung des Hirntods. Dritte Fortschreibung 1997 mit Ergänzungen gemäß Transplantationsgesetz (TPG). Dt Ärztebl 1998 A 1861 ff.; Förderreuther/Angstwurm a. a. O., S. 672 ff.; Haupt, a. a. O. Rn. 16; Link/Rohling/Wagner/Schulz, Die klinische Feststellung des Hirntodes - Eine Video Produktion, 1991; Sinner/Graf a. a. O. S. 497 ff.; Spittler a. a. O. S. 15 ff.

76 Höchst fraglich, aber im Rahmen dieser Arbeit nicht zu vertiefen, ob allein die Befassung des Wissenschaftlichen Beirats der Bundesärztekammer zur Erstellung der Richtlinien zur Feststellung des Hirntodes und deren Bekanntmachung einerseits dem Parlamentsvorbehalts und den Wesentlichkeitsgrundsätzen entspricht und andererseits das gesetzlich berufene Organ darstellt.

77 Wissenschaftlicher Beirat der Bundesärztekammer, a. a. O. S. A-1861 ff., siehe auch Bajanowski/Brinkmann, a. a. O., S. 17 f.; Spittler a. a. O. S. 15 ff.

78 Madea/Henssge/Dettmeyer a. a. O. S. 163; Zippelius, Forum: An den Grenzen des Rechts auf Leben, JuS 1983, 659 ff. 
Menschsein das Einsetzen der Eröffnungswehen der Mutter und ein zu diesem Zeitpunkt tatsächlich lebendes Kind entscheidend ${ }^{80}$. Zudem wird das ungeborene menschliche Leben dem grund- und strafrechtlichen Schutz ab dem Zeitpunkt der Nidation der befruchteten Eizelle unterstellt ${ }^{81}$.

Unumstritten steht zum Lebensende des Menschen derzeit fest, dass Sterben als ein Prozess zu betrachten ist, in dessen Verlauf Organe zu unterschiedlichen Zeitpunkten ausfallen und absterben ${ }^{82}$. Wie die Geburt stellt auch das Sterben einen zeitlich unterschiedlich langen Verlaufsprozess dar, der durch den Tod beendet wird. Normativ werden z. B. aus dem Schwangerschafts- und Geburtsprozess gewillkürte Zeitpunkte herausgegriffen, um den Beginn des menschlichen Lebens und des Menschseins als Individuum zu definieren. Für den Todesbegriff wird deshalb teilweise ebenfalls ein Dualismus vorgeschlagen, um auf unterschiedliche Rechtskonstellationen und von einander abweichende Zwecke reagieren zu können. Dabei wird eine Zweiteilung des Todesbegriffs bevorzugt, der sich an der Zweckbeziehung des jeweiligen Rechtsinstituts orientiert. Für Fragen der Transplantation und Beendigung der Reanimation bei künstlicher maschineller Aufrechterhaltung von Atmung und Kreislauf soll dann auf den Gesamthirntod und bei Rechtsproblemen der Versorgung und Versicherung sowie bei familien- und erbrechtlichen Konstellationen auf den klassischen Herz-Kreislauf-Tod abgestellt werden ${ }^{83}$. Auch im Betreuungsrecht, z. B. bei der Schwangerschaft einer Hirntoten, wird diese Differenzierung empfohlen ${ }^{84}$. Nicht weiter zwischen den Rechtsgebieten des Zivilrechts unterscheidend, wird teilweise beim Einsatz einer Herz-Lungen-Maschine der Hirntod ansonsten der Herz-Kreislauf-Tod befürwortet ${ }^{85}$. Zwei verschiedene Todesdefinitionen werden auch durch den zeitlichen Faktor und die gebotene Eile bei der Transplantation und die fehlende Eilbedürftigkeit bei Fragen des Erbrechts für erforderlich gehalten ${ }^{86}$. Westermann zerlegte das Kriterium des Todes bereits in einen Handlungs- und einen Feststellungsbegriff, wobei für das Handeln, z. B. für Beendigung intensivtherapeutischer Maßnahmen oder den Beginn der Organtransplantation, der Gesamthirntod, und für die Feststellung, z. B. bei erbrechtlichen Konstellationen, der Herz-Kreislauf-Tod maßgeblich sei ${ }^{87}$. Wegen

79 Aufhebung durch Art. 1 Nr. 35 des 6. StRG.

80 BGHSt 32, 194.

81 BVerfGE 39, 1 ff.; 88, 203 ff.

82 Vultejus, a. a. O. 435 (436); Wagner/Brocker, Hirntodkriterium und Lebensgrundrecht, ZRP 1996, 226 (226).

83 Deutsch, a. a. O. 1999, Rn. 405 a; Leipold, Erbrecht, 15. Aufl. 2004, § 2 Rn. 31; Schönig, Zur Feststellung des Todeszeitpunktes, NJW 1968, 189 (190).

84 Kern, Anmerkungen: Zum Todeszeitpunkt des BGB, MedR 1993, 112 (113).

85 Jauernig, BGB-Kommentar, 11. Aufl. 2004, § 1 Rn. 3.

86 Medicus AT-BGB, 8. Aufl. 2002, Rn. 1052: Widersprüchlich stellt er aber einerseits auf den Gehirntod als Ende der Gehirntätigkeit und den Gesamthirntod unter Verweis auf das OLG Frankfurt ab. Der Gehirntod wird im deutschen Rechtsraum als Gesamthirntod und nicht als Teilhirntod verstanden.

87 Westermann H., Fortschritte der Medizin und die Grenzen der ärztlichen Pflicht, in: Jahresschrift der Gesellschaft zur Förderung der Westfälischen Wilhelms-Universität zu Münster, 1968, S. 89; Westermann H. P., Grundbegriffe des BGB, 15. Aufl. 1999, S. 18 mit Hinweis auf einzelfallbezogene Entscheidung; derselbe in Erman/Westermann, BGB, 11. Aufl. 2004, § 1 Rn. 5 mit einem Abstellen auf den Hirntod, wenn er auf einer ärztlichen Feststellung beruht. 
der Manipulationsmöglichkeiten durch Hinauszögern des Herz-Kreislauf-Todes ${ }^{88}$, der Unsicherheiten der Differenzierung ${ }^{89}$ sowie der Einheit der Rechtsordnung ${ }^{90}$ wird sich gegen eine Zweiteilung des Todesbegriffs ausgesprochen. Aus rechtsmedizinischer Erfahrung und pragmatischen Gesichtspunkten wird je nach Art des Lebensendes für eine rechtliche Aufspaltung des Todesbegriffes plädiert ${ }^{91}$.

\section{Maßgebliche Todeszeitpunkte in der verfassungsrechtlichen Darstellung}

Innerhalb der grundgesetzlichen Ordnung stellt das Recht auf Leben einen der Höchstwerte dar, dessen Schutz von der Geburt bis zum Tod andauert ${ }^{92}$ und dem Staat grundsätzlich kein Recht über Leben und Tod des Menschen zubilligt ${ }^{93}$. Das Grundgesetz definiert die Begriffe Leben und Tod nicht näher, stellt andererseits das Recht auf Leben unter den Schutz des Art. 2 II 1 GG. In verfassungsrechtlicher Hinsicht kommt dem/den Todeszeitpunkt/en und den Todeskriterien eine zentrale Bedeutung für die Abgrenzung des Schutzgutes Leben zu dem über den Tod hinauswirkendem postmortalen Persönlichkeitsrecht und die entsprechenden Schutzpflichten des Staates zu. Der staatlichen Schutzpflicht unterliegt menschliches Leben in seiner Einzigartigkeit und seinem individuellen Menschsein und nicht allgemein menschliches Leben ${ }^{94}$, wobei nach Ansicht des Bundesverfassungsgerichts im sachlichen Schutzbereich der körperlichen Unversehrtheit der Mensch als »Einheit von Leib, Seele und Geist « zu begreifen ist ${ }^{95}$. Dem grundrechtlichen Lebensschutz wird das körperliche Dasein als biologisch-physische Existenz unterstellt ${ }^{96}$. Die herrschende Auffassung in der verfassungsrechtlichen Literatur sieht das Recht auf Leben bzw. das menschliche Leben mit dem Ganzhirntod als beendet an ${ }^{97}$. Heun wertet bei intensiv-medizinischer Aufrechterhaltung der Herz-Kreislauffunktion und Atmung den Hirntod, ansonsten den Herzund Kreislaufstillstand als Lebensgrenze ${ }^{98}$. Kunig führt aus, dass Menschsein und Würdeschutz mit dem Leben beginnen, aber nicht sogleich mit dem Tod erlöschen.

88 Rüthers/Stadler, BGB-AT, 11. Aufl. 2001, § 14 Rn. 12.

89 AK-BGB-Kohl, 1987, § 1 Rn. 14.

90 Vgl. befürwortende Nachweise bei Leipold in MünchKommBGB, 3. Aufl. 1997, § 1922 Rn. 12 a Fn. 9.

91 Madea/Dettmeyer/Schmidt, a. a. O. S. 25 ; Madea/Henssge/Dettmeyer a. a. O. S. 163.

92 BVerfGE 39, 1 (42); 49, 24 (53).

93 Kannengießer in Schmidt-Bleibtreu/Klein (Hrsg.) Kommentar zum Grundgesetz, 9. Aufl. 1999, Art. 2 Rn. 20 a f.

94 BVerfGE 88, 203 (252) = NJW 1993, 1751 (1753).

95 BVerfGE 56, 54 (75).

96 Epping, Grundrechte, 2004 S. 38; Jarass in Jarass/Pieroth, Grundgesetzkommentar, 7. Aufl. 2004, Art. 2 Rn. 61 ff.: Widersprüchlich sind insoweit Ausführungen, die den Schutz des Grundrechts auf das körperliche Dasein als die biologisch-physische Existenz unter Ablehnung lebensunwerten Lebens beziehen, gleichfalls aber die Grundrechtsberechtigung mit dem Erlöschen der Hirnströme also dem Ende der biologisch-psychischen bei noch bestehender biologisch-physischer Existenz negieren.

97 Anderheiden a. a. O. S. 371 ff.; Starck in Starck (Hrsg.), Das Bonner Grundgesetz, 4. Aufl. 1999, Art. 2 Abs. 2 Rn. 176; Wiedemann in Umbach/Clemens (Hrsg.) Grundgesetz, 2002, Art. 2 Rn. 295 ff.

98 Heun, a. a. O., S. 217. 
Den Tod als Ende der eigenen Grundrechtspersönlichkeit des Menschen sieht er im Zeitpunkt des endgültigen Erlöschens der Hirnströme für gegeben ${ }^{99}$. Im Zweifel wird sich für die das Schutzgut Leben umfassendere Auslegungsmöglichkeit ausgesprochen und zugunsten des Todeskriteriums, das den »Tod erst bei völligem Zusammenbruch des gesamten Organismus « definiert, plädiert ${ }^{100}$. Andererseits wird darauf verwiesen, dass dem Grundgesetz kein Lebensschutz bis zum biologischen Tod des Menschen zu entnehmen sei, sondern insbesondere im Kontext des Todes der Auslegungsgrundsatz »in dubio pro vita « keine Rolle spiele ${ }^{101}$. Weitergehend wird der Schutz des menschlichen Lebens von der Geburt bis zum klinischen Tod gefasst ${ }^{102}$. Aus verfassungsrechtlicher Sicht werden zudem erhebliche Bedenken gegen die gesetzgeberische Übertragung der Richtlinienkompetenz zur Hirntodfeststellung auf die Bundesärztekammer erhoben, wobei die Vorbehalte mit der fehlenden demokratischen Legitimation der Bundesärztekammer, zu weitgehenden Ausformulierungen der Richtlinien, verfassungsrechtlichen Aspekten der Wesentlichkeitsgrundsätze und des Parlamentsvorbehalts begründet werden ${ }^{103}$.

\section{Maßgebliche Todeszeitpunkte in der transplantationsrechtlichen Erörterung}

Bei der Erörterung und vor der Verabschiedung des Transplantationsrechtes hat sich die Diskussion um den Tod und seine Feststellung auf breiter Ebene kontrovers entzündet und ist bis heute nicht verstummt. Die Vorgaben des TPG und die Kritikpunkte gegen das Gesetz haben in den letzten Jahren entscheidend die Debatte um die Begriffe Sterben, Tod und Todesfeststellung bestimmt. Im Hinblick auf die unzureichende Spendersituation, lange Wartelisten, Wartezeiten der Patienten von vielen Jahren auf ein passendes Organ, gesundheitliche Verschlechterung der Patienten auf den Wartelisten, Versterben von Patienten auf der Warteliste usw. ist in der öffentlichen Diskussion insbesondere um den Hirntod einerseits Sensibilität im Umgang mit Argumenten angesagt, um potentielle Organspender nicht durch das Schüren irrationaler Ängste zu verschrecken, andererseits aber auch eine Offenheit im Umgang mit Argumenten erforderlich, die sowohl dem Selbstbestimmungsrecht des aufgeklärten und informierten Menschen Rechnung trägt als auch den Transplantationspragmatismus im Alltag der kritischen Würdigung unterwirft. Als ethisches Grundprinzip unserer Gesellschaft gilt unzweifelhaft, dass menschliches Leben nicht für den Preis der Rettung eines anderen Menschenlebens geopfert werden darf ${ }^{104}$. Die Gratwanderung für gesetzliche Regelungen in der Transplantationsmedizin wurde daher schon dahinge-

99 Kunig in von Münch/Kunig: Grundgesetzkommentar 5. Aufl. 2000, Art. 1 Rn. 14.

100 Murswiek in Sachs: Grundgesetzkommentar, 3. Aufl. 2003, Art. 2 Rn. 142.

101 MünchKommStraf/Schneider Vor $§ 211$ ff. Rn. 26.

102 Model/Müller, Grundgesetz, 11. Aufl. 1996, Art. 2 Rn. 20, allerdings mit anschließender Relativierung, ob Leben nicht von der Menschenwürde auszulegen sei und reine Vegetationsformen, deren überwiegende Lebensformen durch Maschinen ersetzt werden, noch als Leben zu werten seien.

103 Erman/Schlüter, BGB, 11. Aufl. 2004, § 1922 Rn. 2; Höfling/Rixen a. a. O. § 3 Rn. 9,13 ff.

104 Wolfslast, Grenzen der Organgewinnung - Zur Frage einer Änderung der Hirntodkriterien, MedR 1989, 163 (164). 
hend beschrieben, dass »der Spender so tot wie möglich, das Spendeorgan so lebendig

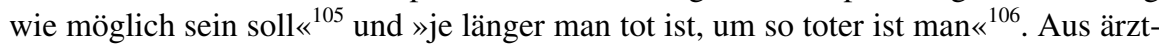
licher Sicht ergibt sich das Dilemma der widerstreitenden Interessen des sterbenden Organspenders mit dem intensivmedizinischen Organschutz für die in Erwägung zu ziehende Explantation zu Gunsten eines überwiegend dem behandelnden Arzt persönlich gar nicht bekannten Organempfängers ${ }^{107}$.

Nach dem Wortlaut des TPG hat der Gesetzgeber zwei Todesbegriffe für die Todesfeststellung vorgegeben ${ }^{108}$. Während in $\S 3$ Abs. 2 Nr. 2 TPG auf das Kriterium des Gesamthirntodes als eine Zulässigkeitsbedingung für die Organentnahme abgestellt wird, sieht $\S 5$ Abs. 1 S. 2 TPG als weiteren Todesbegriff noch den endgültigen, nicht behebbaren Stillstand von Herz und Kreislauf bei einem Zeitablauf von mehr als drei Stunden vor. Insgesamt trifft das TPG aber keine gesetzliche Regelung zum Todeszeitpunkt, sondern stellt lediglich ein Zulässigkeitskriterium für die Organentnahme dar bzw. verbietet eine Organentnahme - mit Ausnahme der Lebendspende gemäß $\S 8$ TPG - vor dem Hirntod ${ }^{109}$.

Überwiegend wird der Gesamthirntod als Explantationskriterium bzw. Individualtod des Menschen anerkannt, der die Billigung des Gesetzgebers im Transplantationsgesetz gefunden hat, auch wenn noch kein Herzkreislaufstillstand eingetreten ist ${ }^{110}$. Andererseits wird der Hirntote teilweise als lebendig und nicht als tot gewertet, so dass Hirntod nicht mit dem Individualtod gleichgesetzt werden dürfe ${ }^{111}$. Nach dieser Auffassung tritt der Tod des Menschen erst mit dem weiteren Herzkreislaufstillstand nach dem Hirntod ein. Die Hirntodkritiker bezweifeln, dass das individuelle Menschsein an der Gehirnfunktion festgemacht werden kann und lehnen den Ganzhirntod als unsicheres Zeichen für das Ende der leib-seelischen biologischen Einheit des Organismus ab. Deshalb wird von Hirntodgegnern sogar teilweise nur die Lebendspende als rechtskonform betrachtet ${ }^{112}$.

105 Vgl. Jauernig a. a. O.; Rüthers/Stadler, a. a. O.; Schettler u. a., Stud. Gen. 1970, S. 304.

106 Geilen, a. a. O. 375.

107 Jähnke a. a. O. Rn. 10.

108 Str. siehe Parzeller/Henze/Bratzke, Gewebe- und Organtransplantation - Verfehlte und praxisferne Regelungen im Transplantationsgesetz, KritV 2004, S. 371 ff. m. w. N.

109 Merkel, Hirntod und kein Ende, Jura 1999, S. 113 (115).

110 Heuer, Conrads, Aktueller Stand der Transplantationsgesetzgebung, MedR 1997, 195 (197); Heun, Der Hirntod als Kriterium des Todes des Menschen - Verfassungsrechtliche Grundlagen und Konsequenzen, JZ 1996, 213; Nickel/Schmidt-Preisigke/Sengler, Kommentar zum Transplantationsgesetz, 2001, § 3 Rn. 7 ff.; Taupitz, Um Leben und Tod: Die Diskussion um ein Transplantationsgesetz, JuS 1997, 203 (207); Uhlenbruck/Ulsenheimer, Die zivilrechtliche Problematik der Organtransplantation, in Laufs/Uhlenbruck (Hrsg.) Handbuch des Arztrechts, 3. Aufl. 2002, § 131 Rn. 8; Vultejus, a. a. O. 435 (438).

111 Beckmann, ZRP 1996, 219 ff.; Höfling, Um Leben und Tod: Transplantationsgesetzgebung und Grundrecht auf Leben, JZ 1995, 26; Geilen a. a. O. S. 393; Höfling/Rixen a. a. O. $\S 3$ Rn. 7 ff.; Grewel, Zwischen Lebensrettung und Euthanasie - das tödliche Dilemma der Transplantationsmedizin, ZRP 1995, 217; In der Schmitten, in Höfling (Hrsg.) Transplantationsgesetz, Anhang zu § 3 Rn. 36 ff. Rixen, 1995 a. a. O. S. 466; Wagner/Brocker, Hirntodkriterium und Lebensgrundrecht, ZRP 1996, 226 (226).

112 Beckmann ZRP 1996, 219. 
Eine Organentnahme bei anenzephalen Neugeborenen vor dem Eintritt des irreversiblen Gesamthirntodes und die Gleichsetzung dieses Zustandes mit dem Gesamthirntod aus pragmatischen Gründen der Organgewinnung wird überwiegend $u$. a. wegen der unzulässigen Relativierung des Lebensschutzes und dem Vertrauensverlust der Öffentlichkeit in die Transplantationsmedizin bei einem zu zweckorientierten Pragmatismus bei der Organgewinnung abgelehnt ${ }^{113}$. Vereinzelt werden durch Abkehr vom Gesamthirntod zum Kortikaltod, also dem alleinigen Ausfall des Großhirns bei intaktem Klein- und Stammhirn, der Anenzephalus als Totgeburt und der Apalliker als Leichnam gewertet ${ }^{114}$. Eine gesetzliche Regelung eines wie auch immer gearteten Teilhirnkonzepts wäre aber als Verstoß gegen die Menschenwürdegarantie des Art. 1 I GG und das Lebensgrundrecht des Art. 2 II GG anzusehen ${ }^{115}$.

\section{Maßgebliche Todeszeitpunkte in der zivilrechtlichen Darstellung}

In unterschiedlichen rechtlichen Konstellationen (z. B. Erbrecht, PStG, VerschG) kann es erforderlich werden, auf einen konkreten Zeitpunkt abzustellen, um z. B. den Übergang des Vermögens im Wege der Erbfolge bestimmen zu können. Wie bereits ausgeführt, wurde das Ende der Rechtsfähigkeit des menschlichen Individuums im BGB nicht normiert und definiert. $§ 1,1922$ BGB setzen das Ende der Rechtsfähigkeit des Menschen mit dessen Tod aber voraus.

Allgemein wird definiert, dass »Tod kein punktgenaues Ereignis, sondern als Sterben ein Vorgang in der Zeit ist ${ }^{116}$. Damit werden die Begrifflichkeiten Sterbeprozess und Tod quasi gleich gesetzt, was eine rechtliche Wertung zunächst nicht erleichtert, da es in manchen Fallkonstellationen (z. B. Versterben von mehreren Familienmitgliedern bei einem Autounfall) wegen der erbrechtlichen Folgen auf eine exakte Bestimmung eines definierten Todeszeitpunktes ankommen kann. Deshalb stellt sich für die Gesamtrechtsnachfolge gemäß $§ 1922$ BGB die Frage, auf welche Form bzw. Feststellung des Todes es im Zweifelsfall ankommt. Das OLG Köln verweist zwar auf die mitunter bestehende Schwierigkeit zur Feststellung des exakten Todeszeitpunkts, spricht sich aber für einen konkreten Todeszeitpunkt durch den sicheren Eintritt des Hirntodes und gegen einen Sterbezeitraum aus. Nach der herrschenden Auffassung im Zivilrecht wird ebenfalls das Gesamthirntodkonzept bevorzugt ${ }^{117}$. Leipold differen-

113 Kloth, a. a. O. S. 188; Isemer/Lilie, Rechtsprobleme bei Anencephalen, MedR 1988, S. 66 (69); Wolfslast, a. a. O. $163 \mathrm{ff}$.

114 Funck, Der Todeszeitpunkt als Rechtsbegriff, MedR 1992, S. 182 (188) der das apallische Syndrom mit einem vollständigen Untergang der Großhirnrinde gleichsetzt, was entgegen der medizinischen Auffassung bei Spittler a. a. O. S. 80 bei weitgehender Erhaltung anderer Hirnfunktionen extrem selten ist.

115 Wagner/Brocker a. a. O. 226 (230).

116 Bamberger in Bamberger/Roth, BGB, 2003, § 1 Rn. 17.

117 AG Hersbruck, MedR 1993, 111 zur Betreuung einer hirntoten Schwangeren: »Die Betroffene ist tot im Sinne des Gesetzes«; BayObLG a. a. O.: »Der Mensch ist tot, wenn eine Reanimation ausgeschlossen ist und wegen eines vollständigen und irreversiblen Funktionsverlustes des Gehirns dauernd keine Gehirnkurven mehr geschrieben werden können.« OLG Köln a. a. O.: »Todeszeitpunkt i. S. der $\S$ 1922, 1923 BGB ist der Eintritt des Hirntodes im Sinne eines irreversiblen Funktionsverlustes des Gehirns, so dass dauerhaft keine Gehirnkurven 
ziert dahingehend, dass aus Gründen der Einheit der Rechtsordnung (ansonsten Diskrepanz zur straf- und arztrechtlichen Beurteilung) auch im Zivilrecht, insbesondere dem Erbrecht, der Gesamthirntod als maßgebliches Kriterium heranzuziehen ist, bei Zweifeln aber auf die Beendigung der Atmungs- und Kreislauftätigkeit abzustellen $\operatorname{sei}^{118}$. Teilweise wird in der Literatur gefordert, im Zivilrecht allein den endgültigen Stillstand des Herz-Kreislaufs zur Todesfeststellung heranzuziehen und sich allein mit dem Feststellungsbegriff Westermanns zu begnügen ${ }^{119}$. In der erbrechtlichen Literatur wird vertreten, dass auch beim Hirntod der wirkliche Zeitpunkt des Todes nicht exakt festgestellt werden kann, sondern der dokumentierte Zeitpunkt der abschließenden Untersuchung als gewillkürter Wert gewählt wird, so dass aus Gründen der Rechtssicherheit im allgemeinen nicht der Gesamthirntod sondern der Herz-Kreislauf-Tod zu bevorzugen sei ${ }^{120}$. Wolf spricht sich aus Gründen der Rechtssicherheit dafür aus, den Tod anzunehmen, wenn »sowohl Herz- als auch Hirntod eingetreten ist ${ }^{121}$. Ebenfalls aus Gründen der Rechtssicherheit plädieren Medicus und Dörner für den Eintritt des Erbfalls und den Verlust der Rechtsfähigkeit des Sterbenden, bei Divergenz der Zeitpunkte, auf den jeweils letzten maßgeblichen Todeszeitpunkt (entweder Hirntod oder klinischer Tod) abzustellen ${ }^{122}$. Bei Todeseintritt unter ärztlicher Betreuung werden zur exakten Bestimmung des Todeszeitpunktes beim Herz-Kreislauf-Tod eine zusätzliche

mehr mitgeschrieben werden können und eine Reanimation ausgeschlossen ist.« OLG Frankfurt NJW 1997, 3099: »Im Erbrecht ist als Todeszeitpunkt der Eintritt des Gesamthirntodes zu verstehen.« Die oberlandesgerichtlichen Entscheidungen werten zwar nach ihrem Gesamtkontext den Gesamthirntod als maßgeblichen Todeszeitpunkt, sind jedoch von ihren Ausführungen etwas missverständlich bzw. erläuterungsbedürftig. Eine Reanimation, wie sie üblicherweise im Notfalleinsatz ohne die Möglichkeit der Hirntoddiagnostik durchgeführt wird, kann bei einem schon hirntoten Patienten durchaus als kardio-pulmonale Reanimation nicht aber als cerebrale Reanimation »erfolgreich« sein. Der Reanimationsbegriff ist daher weiter auszulegen, indem er die Irreversibilität der Wiederbelebung des Gehirns beschreibt. Das OLG Frankfurt differenziert missverständlich zwischen dem »Ausfall der Gesamtfunktion des Gehirns« und »völligem Ausfall auch des Hirnstamms«, der beim Gesamthirntod üblicherweise konzeptionell enthalten ist. Bamberger, a. a. O., Rn. 5; Edenhofer in Palandt, BGB, 63. Aufl. 2004, § 1922 Rn. 2; Heinrichs in Palandt, BGB, 63. Aufl. 2004, § 1 Rn. 3; AK-BGB-Kohl, a. a. O., Rn. 14; MünchKommBGB/Schmitt, 4. Aufl. 2001, § 1 Rn. 22; Soergel/Stein, a. a. O., Rn. 3; Hk-BGB/Staudinger, 3. Aufl. 2003, § 823 Rn. 2.

118 Leipold, 1997 a. a. O.

119 Staudinger/Weick/Habermann, 1995, Vorbem zu § 1 VerschG.

120 Lange/Kuchinke, a. a. O.

121 Wolf in Larenz/Wolf Allgemeiner Teil des BGB, 9. Aufl. $2004 \S 5$ Rn. 24.

122 Hk-BGB/Dörner, 3. Aufl. 2003, § 1 Rn. 5; Medicus, a. a. O. Rn. 1052: «Im Hinblick auf Rechtsfähigkeit und Erbfolge ist daher für den Tod von den in Betracht kommenden Zeitpunkten der letzte zu wählen. Dann gibt es keine Wiederbelebung nach einem vorangegangenen Tod: Rechtlich hat dann nur ein Scheintod vorgelegen.« Hierbei ist zu ergänzen, dass diese Ausführungen zum Scheintod missverständlich sind. Bei einem vorausgegangenen Hirntod können durchaus erfolgreich Herz, Kreislauf und Atmung reanimiert werden. Eine Wiederbelebung des Gehirns ist bei dem Gesamthirntod nicht mehr möglich. Es liegt in diesen Konstellationen also der Gesamthirntod vor, der nicht mit einem auch auf die Gehirnfunktion möglicherweise reversiblen Scheintod gleichgesetzt werden kann. 
Sterbezeit von 10 Minuten und beim Gehirntod der Nachweis des zerebralen Kreislaufstillstandes und eine zeitliche Sicherheitsspanne gefordert ${ }^{123}$.

\section{Maßgebliche Todeszeitpunkte in der strafrechtlichen Darstellung}

Das Strafgesetzbuch enthält weder eine Definition des Todesbegriffs noch gibt es Auskunft über Kriterien der Todesfeststellung. Der strafrechtliche Schutz menschlichen Lebens dauert bis zum Tod des Individuums und umfasst den Todgeweihten ${ }^{124}$ sowie den Sterbevorgang inklusive der Phase der Agonie ${ }^{125}$. Aus strafrechtlicher Sicht würde eine Ablehnung des Hirntodkonzepts im Transplantationsrecht zu erheblichen mit der derzeitigen Gesetzeslage nicht zu vereinbarenden Wertungswidersprüchen führen. Die Explantation bei einem dann noch als lebenden Menschen zu betrachtenden Hirntoten würde einen letalen Eingriff beim Organspender darstellen, was selbst bei Zustimmung des Organspenders als Tötung auf Verlangen gemäß § 216 StGB strafrechtlich gewertet werden könne ${ }^{126}$. Bei einer Ablehnung des Hirntodes als Tod des Menschen wäre die vorsätzliche und gezielte Herbeiführung des Hirn- aber nicht des Individualtodes durch den Täter nach einer Auffassung dann auch nicht als Totschlag oder Mord zu werten ${ }^{127}$.

Die überwiegende Ansicht in der strafrechtlichen Literatur spricht sich für einen einheitlichen Todesbegriff in Form des Hirntods als feststellbaren Todeszeitpunkt des Menschen und die Vorverlagerung vom klinischen Tod auf den Hirntod als strafrechtlichen Todeszeitpunkt aus ${ }^{128}$. Einzelne Auffassungen widersprechen der herrschenden Ansicht, indem sie den Zeitpunkt des Todes vorverlagern oder dem Hirntodkonzept als Ganzes kritisch gegenüber stehen. Eher zweifelnd äußert sich Horn mit seinen

123 Englert, Todesbegriff und Leichnam als Element des Totenrechts, 1979, S. 72 ff. MüllerChristmann in Bamberger/Roth, BGB, 2003, § 1922 Rn. 5; Soergel/Stein a. a. O. Rn. 4; mit Ablehnung bei Lange/Kuchinke, Erbrecht, 5. Aufl. 2001, S. 76 Fußnote 6.

124 Maurach/Schroeder/Maiwald, Strafrecht-BT, 9. Aufl. 2003, § 1 IV Rn. 11; OGH 2, 140.

125 Maurach/Schroeder/Maiwald, a. a. O.; BGHSt 7, 288; BGH VRS 17, 187; BayObLG NJW $1973,565$.

126 Heun a. a. O. S. 218; Merkel a. a. O. S. 121 verweist zu Recht darauf, dass eine solche Wertung das Ende der postmortalen Transplantationsmedizin bedeuten könnte.

127 Herrig, a. a. O. ,S. 91.

128 Brodag, Strafrecht BT, 8. Aufl. 1998, § 211 Rn. 3; Eser in Schönke/Schröder, Strafgesetzbuch, 26. Aufl. 2001, §§ 211 ff. Vorbem. Rn. 19; Fischer in Fischer/Tröndle 52. Aufl. 2004, Vor $\S \S 211$ bis 216 Rn. 8; Imbach, Der Gesamthirntod als Kriterium des Todes und die Kritik daran, in Roxin/Schroth (Hrsg.) Medizinstrafrecht - Im Spannungsfeld von Medizin, Ethik, Strafrecht, 2000, S. 189 ff.; Kindhäuser, Strafgesetzbuch, 2002, Vor § 211 Rn. 3; Krey, Strafrecht-BT, Rn. 17 unter Hinweis auf die h. M. aber auch auf die »beachtlichen Bedenken bei Geilen«; Kühl in Lackner/Kühl StGB, 25 Aufl. 2004, Vor § 211 Rn. 4; Jähnke in LK, 11. Aufl. 2002, Vor § 211 Rn. 9; Joecks, Strafgesetzbuch, 4. Aufl. 2003, Vor $\S 211$ Rn. 25; MünchKommStGB/Schneider, 2003, Vor $§ 211$ ff. Rn. 15; Otto, Grundkurs Strafrecht, 6. Aufl. 2002, § 2 Rn. 10: Mit einer missverständlichen Darstellung (»irreversibler Zusammenbruch des Gehirns bei noch aufrechterhaltener Kreislauffunktion im übrigen Körper «), was zwar den Zustand auf einer Intensivstation beschreibt, aber nicht den Hirntod bei bereits zuvor eingetretenem klinischen Tod; Wessels/Hettinger, Strafrecht Besonderer Teil 1, 27. Auflage 2003, Rn. 21. 
Ausführungen, dass »man heute glaubt als Zentrum des menschlichen Lebens das Gehirn gefunden zu haben ${ }^{129}$. Tröndle führt aus, dass der Gesetzgeber außerhalb seiner Kompetenz und Legitimation den Hirntod als Ganzheitstod definiert habe und wertet den Sterbeprozess eines Hirntoten als grundrechtlich zu schützenden Bereich eines noch lebenden Sterbenden ${ }^{130}$. Gropp verweist auf die Interessengewichtung zwischen Organspender und Empfänger als primäre Aufgabe des Hirntodkriteriums ${ }^{131}$. Teilweise wird im Abstellen auf den Hirntod an Stelle des klinischen Todes sogar eine Übergewichtung des Transplantationsinteresses und eine Unterbewertung des Lebenserhaltungsinteresses des Kranken gesehen ${ }^{132}$. Vereinzelt wird aus strafrechtlicher Sicht aus dem Prinzip des Lebensschutzes primär eine Lebendspende in Betracht gezogen, weil das »Ende der leib-seelischen Einheit und der biologischen Einheit des Organismus als Ganzem durch den Gesamthirntod nicht angezeigt werden « könne ${ }^{133}$. Dencker spricht sich im Strafrecht für einen vorzeitigen Todesbegriff aus, der schon mit dem alsbaldigen Eintritt des Hirntodes als »endgültiges Abschneiden einer Chance zu leben « beginnt und bereits bei irreversibler Bewusstlosigkeit bei irreversibler Hirnschädigung wenige Stunden vor dem Gesamthirntod vorliegen soll ${ }^{134}$. Dieses zeitlich dem Hirntod vorgelagerte Konzept wird wegen seiner mangelnden Bestimmtheit, unklaren Grenzziehung, wertenden Betrachtungen sowie unsicheren Prognosen abgelehnt ${ }^{135}$. Eine Reduzierung des Gesamthirntods auf Teilhirnkonzepte zur Erleichterung von Transplantationen, z. B. Organentnahme bei Anenzephalen, wird in der strafrechtlichen Literatur als nicht vertretbare Relativierung des Lebensschutzes überwiegend abgelehnt ${ }^{136}$. Bei der passiven Sterbehilfe wird zwischen Sterbehilfe im engeren, also wenn der Sterbevorgang bereits eingesetzt hat ${ }^{137}$, und im weiteren Sinn, wenn der Prozess des Sterbens gerade noch nicht eingesetzt hat, unterschieden. In diesem Zusammenhang wird ebenfalls deutlich, wie schwierig eine Abgrenzung im konkreten Einzelfall des Sterbeprozesses werden kann.

129 Horn in SK, 7. Aufl. 2003, § 212 Rn. 5.

130 Tröndle in Tröndle/Fischer, StGB, 49. Aufl. 1999, § 168 Rn. 4 b ff.

131 MünchKommStGB/Gropp § 218 Rn. 8.

132 Joecks, a. a. O. Rn. 24.

133 Beckmann a. a. O. S. 225.

134 Dencker, NStZ 1992, 311 (315): »Tod »ist« nicht der Hirntod, sondern das endgültige Abschneiden einer »Chance zu leben.... Der tatbestandliche Erfolg der Tötungsdelikte ist jedenfalls ... eingetreten, wenn folgende Bedingungen erfüllt sind: a) irreversible Bewusstlosigkeit aufgrund b) irreversibler schwerer Hirnschädigung, die c) zum »alsbaldigen« (nach der Entscheidung: spätestens in »wenigen Stunden« eintretenden) Stillstand aller Hirntätigkeit führen wird.«.

135 Imbach a. a. O. S. 213; Joerden, Tod schon bei »alsbaldigem« Eintritt des Hirntodes? Anmerkungen zu einer These von Dencker, NStZ 1993, S. 268 ff.

136 Kühl, a. a. O.; Otto, a. a. O., Rn. 12; Maurach/Schroeder/Maiwald, a. a. O. Rn. 12; a. A. Funck a. a. O.; Brodag a. a. O.; Hiersche MedR 1984, S. 215; Jähnke LK 218 Rn. 4.

137 BGHSt 40, S. 257 (259 f.); Bernsmann, Der Umgang mit irreversibel bewusstlosen Personen und das Strafrecht, ZRP 1996, S. 87 ff. 


\section{Feststellung des Hirntods aus juristischer Sicht}

Bei der Auswertung der juristischen Literatur und Rechtsprechung ist auffällig, dass die Darstellung der ärztlichen Hirntoddiagnostik häufig in Abweichung von den medizinischen Richtlinien wiedergegeben wird, obwohl teilweise sogar auf diese verwiesen wird. Erläuternd sei deshalb noch einmal dargestellt, dass unabhängig von der Frage der Rechtsverbindlichkeit der Richtlinien des Wissenschaftlichen Beirats der Bundesärztekammer die Feststellung des Hirntodes (z. B. bei krankheitsbedingten Ausnahmen (primär infratentoriellen Prozessen)) nicht an eine apparative Diagnostik geknüpft werden muss, sondern die klinische Befunderhebung (Ausschlussdiagnostik und Erhebung der klinischen Symptome des Ausfalls der Hirnfunktion) sowie die Verlaufsbeobachtung als Irreversibilitätsnachweis durch zwei hinreichend erfahrene und qualifizierte Ärzte für ausreichend betrachtet wird ${ }^{138}$.

Bei der apparativen Diagnostik handelt es sich um ergänzende Untersuchungen, die nur bei bestimmten Krankheits- bzw. Verletzungskonstellationen und bei Kindern bis zum vollendeten 2. Lebensjahr grundsätzlich erforderlich sind oder als Zusatzuntersuchungen zum Ersatz oder zur Verkürzung der Verlaufsbeobachtungen in den zeitlich definierten Abständen herangezogen werden. Eine Durchführung apparativer Diagnostik dient oftmals der Verkürzung der Wartezeit von 12 Stunden $^{139}$. Die ergänzenden diagnostischen Untersuchungen können für sich allein den irreversiblen Hirnfunktionsausfall nicht nachweisen. Die Feststellung des Hirntodes kann deshalb entgegen anderslautenden Darstellungen in der Rechtsprechung und Literatur folglich auf verschiedenen Wegen erfolgen, insbesondere müssen nicht zwingend Hirnströme zur Feststellung des Hirntods abgeleitet werden ${ }^{140}$. Das alleinige Ableiten der Hirnströme mittels eines EEGs kann zu einer fehlerhaften Todesfeststellung selbst bei einem Null-Linien EEG führen, wenn keine Hirnstammschädigung vorliegt ${ }^{141}$.

138 Wissenschaftliche Beirat, 1997 a. a. O. A-1861 ff. »Der Hirntod kann in jeder Intensivstation auch ohne ergänzende apparative Diagnostik festgestellt werden.«; Beck a. a. O.

139 Haupt, a. a. O., Rn. 19; Maurach/Schroeder/Maiwald, a. a. O., Rn. 12.

$140 \mathrm{Vgl}$. die nachfolgenden Angaben, die in ihre Darstellung zu kurz greifen: Bamberger in Bamberger/Roth 2004 a. a. O. »Die Feststellung ist berechtigt, wenn die Hirnstromkurve des EEG eine Nulllinie zeigt«; Brox a. a. O.: »Gehirntod, wenn Hirnströme nicht mehr feststellbar sind «; Fahse a. a. O. § 1 Rn. 12: »... Eintritt des Hirntodes im Sinne eines irreversiblen Funktionsverlustes des Gehirn, so dass dauerhaft keine Gehirnkurven mehr mitgeschrieben werden können « gleichlautend OLG Köln a. a. O. S. 1481; AK-BGB-Kohl, a. a. O. Rn. 13: »Mit der h. M. empfiehlt es sich daher ... auf den Gehirntod - die NullKurve im EEG - abzustellen«; Krey a. a. O.: »Der Nachweis des Gehirntodes soll dabei im Wesentlichen mit dem Auftreten der Nulllinie im EEG erbracht sein.«; Rüthers/Stadler a. a. O. »Null-Linie im EEG«; Schmitt a. a. O.: »Für diesen Bereich setzte sich zunächst die Erkenntnis durch, dass man auf den sog. Hirntod abstellen, d. h. einen Menschen dann als tot ansehen sollte, wenn keine Hirnströme mehr festzustellen sind, also das EEG die sog. Nulllinie verzeichnet.«; Schneider a. a. O.: Rn. 19: »Die zu beachtenden Verfahren sind teilweise derart anspruchsvoll, dass sie selbst in einer Vielzahl modern ausgerüsteter Krankenhäuser derzeit nicht durchgeführt werden können.«

141 Arbeitskreis Organspende, Kein Weg zurück - Informationen zum Hirntod, 2002, S. 16; Forster/Ropohl, a. a. O., S. 9: z. B. bei Hypothermie oder schweren Schlafmittelvergiftungen; Funck a. a. O. S. 187 Fn. 106; Stratenwerth, Zum juristischen Begriff des Todes, in FS Engisch 1969, S. 529 (545). 


\section{Ergebnis}

Im Ergebnis bleibt nach der Analyse der medizinischen und rechtlichen Ansichten und den praktischen Erfahrungen aus der Transplantations- und Rechtsmedizin festzuhalten:

1) Das Sterben des Menschen stellt einen Prozess dar, der durch den Tod des Menschen als irreversiblen point auf no return das Leben des Individuums Mensch beendet (Individualtod) ohne bereits zum Tod menschlichen Lebens im biologischen Sinn (totaler Tod) geführt zu haben.

2) Durch den medizinischen Fortschritt können einzelne Körperfunktionen aufrecht erhalten werden, obwohl lebenswichtige Organe, wie das Gehirn, bereits irreversibel funktionsunfähig sind.

3) Aus rechtlicher und medizinischer Sicht kann es erforderlich werden, im Sterbeprozess den irreversiblen point auf no return möglichst zeitlich genau zu konkretisieren, um z. B. Entscheidungen zur Behandlungsbeendigung, zur Organtransplantation oder $\mathrm{zu}$ vermögensrechtlichen Fragestellungen nach dem Tod treffen $\mathrm{zu}$ können.

4) Durch die Fortschritte der Intensivmedizin hat sich neben dem klassischen Todesbegriff des früher als irreversibel betrachteten Herz- und Kreislauftodes (klinischer Tod: heute reversibel in Abhängigkeit $u$. a. von Grunderkrankung und Reanimation) der Hirntod als irreversibles Erloschensein der Gesamthirnfunktion des Großhirns, des Kleinhirns und des Hirnstammes bei einer intensivmedizinisch noch aufrechterhaltenen Atmung, Herz- und Kreislauffunktion etabliert (dissoziierter Hirntod).

5) Das Kriterium des Gesamthirntods bedeutet nicht, dass sämtliche Funktionen des Gehirns, z. B. auf zellulärer Ebene oder des Zwischenhirns, vollständig erloschen sind.

6) Die herrschende medizinische und rechtliche Auffassung stellt das irreversible Hirnversagen als Gesamthirntod dem Individualtod des Menschen gleich, während eine Mindermeinung den Hirntod als Durchgangsstadium im Sterbeprozess eines noch lebenden Menschen begreift.

7) Der Gesetzgeber hat im Transplantationsgesetz den Gesamthirntod als Mindestvoraussetzung für eine Organentnahme beim dann toten Spender normiert ohne die naturwissenschaftlich-medizinischen Voraussetzungen zu konkretisieren ${ }^{142}$.

8) Entgegen der weitverbreiteten Auffassung bedarf die Feststellung des Gesamthirntodes nicht zwangsläufig der apparativen Diagnostik, z. B. durch Messung der Hirnströme, sondern kann allein durch ärztliche Diagnostik und den zeitlichen Nachweis der Irreversibilität erfolgen.

9) Für die Todesfeststellung kann eine ex-ante-Betrachtung (Untersuchungen vor dem festgestellten Todeseintritt, z. B. Hirntoddiagnostik vor Organentnahme) oder eine ex-post Betrachtung (Untersuchungen nach dem festgestellten Todeseintritt, z. B. Leichenschau) erforderlich sein.

142 Siehe vertiefende Darstellung bei Parzeller/Henze/Bratzke, Gewebe- und Organtransplantation - Verfehlte und praxisferne Regelungen im Transplantationsgesetz, KritV 2004, 371 ff. 
10) Totenflecken, Totenstarre und/oder Fäulnis sind sichere Zeichen des Individualtods und bestätigen indirekt sowohl den irreversiblen Hirn- als auch den irreversiblen Herz-Kreislauftod.

11) Nur bei einem geringen Prozentsatz der Todesfeststellungen, überwiegend bei potentiellen Organspendern, kommt es, um den gesetzlichen Vorgaben des TPG zu entsprechen, auf die Feststellung des Gesamthirntodes mit abgeschlossener Dokumentation der Hirnfunktionsdiagnostik bei intensivmedizinisch erhaltener Atmung und Herz-Kreislauffunktion an. Bei über $99 \%$ der jährlichen Todesfälle erfolgt eine ex-post Feststellung des Individualtodes durch die klassische Leichenschau bei Vorliegen sicherer äußerer Zeichen des Todes oder einer mit dem Leben nicht zu vereinbarenden äußeren Verletzung ohne dass es einer Hirntoddiagnostik bedarf, da beim Vorhandensein von sicheren Zeichen des Todes der Mensch unzweifelhaft tot (individualtot, hirntot, klinisch tot) ist.

12) Jenseits intensivmedizinischer Bedingungen ist eine Feststellung des Gesamthirntodes zur Feststellung des Todes entbehrlich. Die Todesfeststellungen sollten nach den klassischen rechtsmedizinischen Methoden und Berechnungen erfolgen.

13) Für Fragen der Transplantation kann bei erklärtem oder mutmaßlichen Willen des Organspenders auf den Hirntod als Todes- und Feststellungszeitpunkt abgestellt werden, wobei sicherzustellen ist, dass die Bedeutung des Gesamthirntodes auch unter Berücksichtigung der gegensätzlichen Argumente von dem einzelnen Organspender berücksichtigt werden konnte.

14) Im Transplantationsgesetz geht der Gesetzgeber nach dem Wortlaut von einer dualistischen Begrifflichkeit zur Feststellung des Todes in Form des Gesamthirntodes (§ 3 II Nr. 2 TPG) und des endgültig, nicht behebbaren Stillstandes von Herz und Kreislauf über mehr als drei Stunden aus (\$ 5 I 2 TPG).

15) Eine Mehrteilung des Todesbegriffs entsprechend seiner erforderlichen Verwendung ist zu bevorzugen und vom demokratisch legitimierten Gesetzgeber sowohl hinsichtlich der Todesfeststellung als auch des schätzungsweise zu errechnenden Todeszeitpunkts zu konkretisieren.

16) Es bedarf einer gesellschaftlichen Enttabuisierung und einer offenen Diskussion um Sterben und Tod, damit der einzelne in freier Selbstbestimmung sein Lebensende entsprechend seinen individuellen und/oder altruistischen Vorstellungen regeln kann.

17) Die beschriebene Praxis »zur Konditionierung eines postmortalen potenziellen Spenders vor der Multiorganentnahme« ist nicht nur hinsichtlich der Wortwahl fragwürdig.

18) Der Bedarf an Organen und Geweben in Deutschland wird nicht umfassend gedeckt, so dass kranke Patienten zu lange auf Spendeorgane warten, sich ihr Gesundheitszustand zunehmend verschlechtert und die Patienten während der Wartezeit teilweise krankheitsbedingt versterben. 


\section{Fazit}

Eine Antwort auf die Frage, wann ein Mensch im Rahmen des Sterbeprozesses tot ist, bedingt weitreichende persönliche, religiöse, ethische, medizinische und rechtliche Konsequenzen. Personenstandsrechtlich erbringt der Eintrag in das Sterbebuch Beweis für die Tatsache des Todes der eingetragenen Person sowie für den Zeitpunkt des Todes ( $§ 32,60,66,61 \mathrm{a}$ PStG). Rechtlich ist der Mensch zwar zu dem Zeitpunkt tot, der im Leichenschauschein als Todeszeitpunkt vermerkt ist. Jedoch ist der Nachweis der Unrichtigkeit der Beurkundung zulässig ${ }^{143}$.

Anhand der aufgelisteten unterschiedlichen Definitionen zum Tod wurde deutlich, dass der Sterbeprozess verschiedene Begriffe des Todes umfasst und der Begriff $»$ Tod « aufgrund seiner verschiedenen Bedeutungen und Feststellungen auf der Zeitachse des Sterbeprozesses nicht unbedingt zur Definition eines punktuell exakt definierbaren Ereignis greift. Für das Verständnis von Sterben und Tod ist mehr als ein kaum exakt definierbares und messbares Ereignis die Betrachtung des Sterbeprozesses erforderlich, dessen Ergebnis abhängig von unterschiedlichen Einflüssen reversibel oder irreversibel, umkehrbar oder unumkehrbar ist. Nach anthropologischen Kriterien ist beim Sterbeprozess von Bedeutung, ob noch geistig-seelische Fähigkeiten erhalten sind oder ein rein vegetativer Zustand besteht ${ }^{144}$. Gerade die Differenzierung zwischen den beiden letzten Funktionszuständen bestimmt maßgeblich - ohne im Rahmen dieses Beitrages vertiefend auf die Argumente eingehen zu können - die Diskussion von Hirntodbefürwortern und Hirntodgegnern bei der Bewertung des Hirntoten als toten Menschen bzw. als lebenden und sterbenden Menschen ${ }^{145}$, wobei als Konsens beider Auffassungen ein auch durch Wiederbelebungsmaßnahmen nicht mehr überwindbarer, irreversibler und definitiver Funktionsverlust des Gehirns im Zustand des Gehirntodes festzustellen ist ${ }^{146}$. Bei der Nidation kann zunächst menschliches, zelluläres Leben ohne Ausprägung einer subjektiven Individualität mit einer positiven Entwicklungsprognose für subjektive Individualität heranwachsen. Beim hirntoten Patient besteht nach überwiegender Auffassung menschliches Leben nunmehr ohne Ausprägung einer subjektiven Individualität und ohne jegliche positive Entwicklungsprognose für subjektive Individualität für Gegenwart und Zukunft.

Während die Feststellung sicherer Todeszeichen (Totenflecken, Totenstarre und Fäulnis) bzw. Verletzungen, die mit dem Leben nicht vereinbar sind (Dekapitation), keinen naturwissenschaftlich begründeten Zweifel am Ende des menschlichen Sterbevorgangs durch bereits vollzogenen Übergang vom Leben zum Tod aufkommen lässt, unterliegen andere Konzepte der Todesdefinition und -feststellung der kritischen

143 Hübner a. a. O. Rn. 132; Wolf a. a. O. Rn. 28; Weick/Habermann a. a. O. Rn. 10.

144 Vgl. Herrig, a. a. O. S. 85.

145 Beispielhaft aus medizinischer Sicht: Als Hirntodbefürworter Spittler 1998 a. a. O. S. 62: »Im Hirntod ist dieser Mensch, diese individuelle Person nicht mehr«; als Hirntodgegner Bavastro a. a. O. S. 116: »Es handelt sich bei den sogenannten »Hirntoten« biologisch, phänomenologisch und physikalisch um lebende Menschen! Es sind schwerstkranke, sterbende Menschen, ein Sterbender ist aber noch ein Lebender.«

146 Uhlenbruck/Ulsenheimer a. a. O. Rn. 8. 
Betrachtung. In Erweiterung der Richtlinien zur Feststellung des Hirntodes des wissenschaftlichen Beirats ist bei der Feststellung eines äußeren sicheren Zeichens des Todes indirekt nicht nur der Hirn- sondern auch der klinische Tod nachgewiesen. Die Schätzung der Todeszeit kann durch eine nährungsweise Rückrechnung nach rechtsmedizinischen Kriterien erfolgen, indem Ausbildung und Ausprägung früher Leichenzeichen (Totenflecken, Totenstarre), Auftreten supravitaler Leichenzeichen (z. B. mechanische und elektrische Erregbarkeit der Leichenmuskulatur) und die Abnahme der Mastdarmtemperatur nach Todeseintritt unter Berücksichtigung äußerer Faktoren (z. B. Außentemperatur) als Parameter herangezogen werden. Auch die Feststellung des Hirntodes zur Bestimmung des Todeszeitpunktes ist letztendlich eine Schätzung. Mit der Feststellung des Hirntodes ist der irreversible Ausfall der Funktionalität des Gesamthirns bestätigt, dem nach Beendigung intensivmedizinischer Maßnahmen der klinische Tod folgen wird. Eine minutengenaue Bestimmung des tatsächlichen Eintritts des Hirntodes ist reine Fiktion ${ }^{147}$. Nach derzeitiger Definition wird als Todeszeitpunkt des Hirntodes der Abschluss der Diagnose und der Dokumentation gewertet, nicht aber der tatsächliche Eintritt des Hirntodes ${ }^{148}$.

Die Debatte um den Hirntod und auch die mangelnde Spendebereitschaft für Organe und Gewebe zeigen auf, dass es derzeit nicht gelungen ist, der Bevölkerung wesentliche Aspekte der Organspende und deren Voraussetzungen hinreichend transparent zu machen ${ }^{149}$. Ein hinreichender gesellschaftlicher Konsens zur Festlegung des Todes ist, wie auch die unterschiedlichen Auffassungen in der Medizin und im Recht aufweisen, nicht völlig erreicht worden. Eine klare gesetzliche Regelung für alle Rechtsbereiche wäre aus Gründen der Rechtssicherheit aber auch der rechtlichen Überprüfbarkeit wünschenswert. Gegen eine gesetzliche Fixierung des Todeszeitpunktes wird eingewandt, dass die medizinische Forschung bezüglich der Hirntodfeststellung noch zu sehr im Fluss sei ${ }^{150}$. Beck bezweifelt zudem, dass eine gesetzlich klare Festlegung des Hirntodes Zweifel in der Bevölkerung am Tod des hirntoten Angehörigen, dessen Herz noch schlägt, beseitigen kann und fordert eine ehrliche Information der Bevölkerung, die auch das Recht auf Unversehrtheit des Körpers ohne den Aufbau von Schuldgefühlen akzeptiert, da es kein Recht auf fremde Organe gäbe ${ }^{151}$. Ferner wird auf Untersuchungen verwiesen, die Unsicherheiten selbst bei Intensivmedizinern zur klaren Definition des Hirntodes aufweisen ${ }^{152}$. Trotz aller Sachargumente liegt ein

147 Vgl. Funck a. a. O. S. 18; Herrig a. a. O. S. 83; Jähnke a. a. O. Rn. 9: »Eine Bestimmung des Zeitpunkts des Todeseintritts ist überhaupt nicht möglich«; Spittler, a. a. O., 2003, S. 58: »Bis heute gibt es keine praktikable Methode, den Zeitpunkt (besser: den umschriebenen Zeitraum) des Eintritts des Hirntodes zuverlässig zu bestimmen.«

148 Wissenschaftlicher Beirat der Bundesärztekammer, 1998, a. a. O. A-1865: »Todeszeitpunkt: Festgestellt wird nicht der Zeitpunkt des eingetretenen, sondern der Zustand des bereits eingetretenen Todes. Als Todeszeitpunkt wird die Uhrzeit registriert, zu der die Diagnose und Dokumentation des Hirntodes abgeschlossen sind.«

149 Klinkhammer, Transplantation «Das Gesetz muss reanimiert werden«, Dt. Ärztebl. 2002, A-2241 ff.

150 Eser a. a. O. Rn. 20.

151 Beck a. a. O. S. 991.

152 Goetze-Clarén, Der Hirntod ist nicht der Tod des Menschen, Ethik Med 1998, 58 (59). 
»schleichendes Misstrauen in der Bevölkerung gegen die Veränderung der Todesbegriffe $\ll$ vor ${ }^{153}$.

Nach einer Meinungsumfrage des Forschungsinstituts Emnid sind $63 \%$ der Deutschen bereit, nach ihrem Tod Organe für eine Transplantation zur Verfügung zu stellen, jedoch befürchtet jeder dritte Befragte, dass er zu früh für tot erklärt wird, wenn er einen Spendeausweis bei sich trägt ${ }^{154}$. Teilweise wird deshalb argumentiert, dass der Bürger oder Patient durch die öffentliche Diskussion um den Hirntod verunsichert und seine Spendebereitschaft dadurch beeinträchtigt werden könnte ${ }^{155}$. Andererseits belegen Studien, dass die Spendebereitschaft bei einem hinreichenden Verständnis der Angehörigen für das Konzept des Hirntodes und ausreichender Zeit zum Überlegen erhöht war ${ }^{156}$. In § 2 TPG ist eine Aufklärungsverpflichtung spezieller Organisationen vorgesehen, um der Bevölkerung zu Lebzeiten sachgerechte Informationen für ihre persönliche Entscheidung zur Organspende zukommen zu lassen ${ }^{157}$. Eine solche Aufklärung sollte offen erfolgen, damit der potentielle Spender entsprechend seinem Selbstbestimmungsrecht in Kenntnis der relevanten Informationen seine volle Zustimmung zur Organentnahme erteilt, eingeschränkt erteilt (z. B. nur Gewebespende nach dem irreversiblen klinischen Tod) oder verweigert, unabhängig davon ob man die eine oder andere Auffassung zum Hirntod teilt. Rechtshistorisch war der Gesetzgeber schon in der Lage sogar Todesdefinitionen zu schaffen, die den so genannten bürgerlichen Tod als Beerbung bei lebendigem Leib regelten ${ }^{158}$. Ohne Ansehen des tatsächlichen medizinisch-biologischen Zustandes des Menschen wurde der Tod normativ für einen noch Lebenden festgelegt (z. B. Klostertod, Straftod, Siechentod) ${ }^{159}$. Neben der medizinisch-naturwissenschaftlichen Betrachtung ist es deshalb erforderlich einen eindeutigen und klaren gesellschaftlichen Konsens durch den demokratisch legitimierten Gesetzgeber zur Definition des Todes, seiner Festlegung und seines Zeitpunktes oder seiner Zeitpunkte jenseits des Zulässigkeitskriteriums zur Organentnahme zu schaffen ${ }^{160}$.

Wesentliche Aspekte zum Lebensende sind nicht gesetzlich definiert. Definitionsschwierigkeiten können durch eine Vielschichtigkeit von Begriffen und Vorstellungen zum Tod, unterschiedlichen rechtlichen Anforderungen an Todesbegriff und Todesfeststellung aber auch die letztendliche Ungewissheit, was das individuelle Menschsein im Sterbeprozess ausmacht bzw. dieses Menschsein beendet, entstehen. Die Debatte, insbesondere um den Hirntod, ist in ihrer teilweise heftig geführten Argu-

153 Deutsch a. a. O. Rn. 406.

154 Vgl. Management \& Krankenhaus, Zahlen Daten Fakten, 2004, S. 32.

155 Ulsenheimer, Arztstrafrecht in der Praxis, 2003, 3. Aufl. S. 305.

156 vgl. Darstellung amerikanischer Studienergebnisse bei Schulz/Gold/Knesebeck v. d./Koch, Der Organspendeprozess und Ansatzmöglichkeiten zur Erhöhung der Spenderate, Bundesgesundheitsbl - Gesundheitsforsch - Gesundheitsschutz, 2002, S. 774 (777).

157 Uhlenbruck/Ulsenheimer, a. a. O. Rn. 5.

158 Staudinger/Marotzke 1994, § 1922 Rn. 2, 4; Lange/Kuchinke a. a. O. S. 75.

159 Klostertod: Conicilium Tridentinum, sessio XXV. De regularibus et monialibus c. II, in: Corpus Juris Canonici, Pars II, ed. A. L. Richter 1839, S. 86; bürgerlicher Tod im Preußischen ALR. 
mentation nicht frei von persönlichen Angriffen gewesen. Bei dem existenzbeendenden Zeitpunkt des Todes wird jede Wertung, die sich nur auf medizinische, naturwissenschaftliche oder juristische Aspekte zurückzieht, im Vorstellungsbild der Bevölkerung letztendlich versagen. Aus rationalen und irrationalen Ängsten, der Ehrfurcht vor dem Leben aber auch vor dem Tod, der Ebene der Endlichkeit und aus der Hoffnung auf Unsterblichkeit entstehen Fragen, die sich teilweise den naturwissenschaftlichen Denkgesetzen und einer juristischen Rationalität entziehen können.

Unser Verständnis von der Funktion der Organe insbesondere des Gehirns für das Menschsein trägt wesentlich zum Verständnis, wann der Mensch tot ist, bei. Während sich früher in der ethisch-philosophischen Erkenntnis die Frage nach der Leib-SeelenProblematik stellte, ist durch die Ergebnisse der modernen Neurowissenschaften auf eine Gehirn-Seelen-Problematik abzustellen. Das Gehirn als zentrales biologisches Substrat für kognitive und emotionale Prozesse des Menschseins führt dabei zur Frage, ob Menschsein, bzw. das was den Mensch zum Menschen macht, gleichzusetzen ist mit der Funktion seines Gehirns ${ }^{161}$. Je nach ethischem Grundverständnis wird

160 Impuls an die Föderalismus-Kommission:

Gesetzesvorschlag als Gegenstand konkurrierender Gesetzgebung in Erweiterung von Art. 74 I Nr. 26 GG »sowie Regelungen zur Transplantation von Organen und Geweben und zur Definition und Feststellung des Todes des Menschen«: Gesetz zum Tod des Menschen $\S 1$ (Definition des Todes): Der Mensch ist tot, wenn überlebensnotwendige Organe in ihrer Funktion irreversibel und unumkehrbar ausgefallen sind und dieser Funktionsausfall durch zur Verfügung stehende, insbesondere medizinische, Maßnahmen und Mittel, z. B. Transplantationen, nicht mehr behoben werden kann. § 2 (Feststellung des Todes im Sterbeprozess):

1. Der Tod tritt im Verlauf des unumkehrbaren und irreversiblen Sterbeprozesses ein, ohne dass sein zeitlicher Eintritt minutiös vorhergesagt werden kann. Da eine exakte Vorherbestimmung des Todes nicht erfolgen kann, ist der Eintrittszeitpunkt des Todes nachträglich zu bestimmen. Durch den Einsatz intensivmedizinischer Methoden kann die Atmung und die Herz-Kreislauffunktion aufrechterhalten werden, obwohl die Funktion des Gesamthirns (Großhirn, Kleinhirn, Hirnstamm) bereits irreversibel erloschen ist (dissoziierter Hirntod).

2. Zur nachträglichen Feststellung des Todeszeitpunkts ist zwischen zwei Feststellungsverfahren zu unterscheiden: Nr. 1: Die nachträgliche Feststellung des Todeszeitpunkts erfolgt grundsätzlich durch klassische Todesdiagnostik in Form der Feststellung sicherer Zeichen des Todes (Totenflecken, Totenstarre, Fäulnis und/oder mit dem Leben nicht zu vereinbarenden Verletzungen) durch einen amtlichen Leichenschauer. (Verfahren ist im Gesetzestext oder z. B. in einer Anlage zu erläutern). Nr. 2: Die nachträgliche Feststellung des Todeszeitpunkts erfolgt ausnahmsweise unter intensivmedizinischen Bedingungen zum Zweck der Organentnahme durch Feststellung des endgültigen, nicht behebbaren Ausfalls der Gesamtfunktion des Großhirns, des Kleinhirns und Stammhirns entsprechend dem Stand der Erkenntnis der medizinischen Wissenschaft und den Anforderungen von $\S \S 5$ Abs. 1 S. 1 i. V. m. 5 Abs. 2 TPG. Todeszeitpunkt ist der dokumentierte Abschluss der Feststellungsdiagnostik. (Verfahren der Todesfeststellungen ist im Gesetzestext oder z. B. in einer Anlage zu erläutern. Eine Übertragung einer Richtlinienkompetenz auf einen wissenschaftlichen Beirat kann nicht erfolgen).

3. Auch nachträgliche Todesfeststellungen nach Nr. 1 und Nr. 2 können im allgemeinen keinen minutengenauen Todeszeitpunkt belegen, sondern stellen einen normativ bestimmten Todeszeitpunkt im Sterbeprozess dar. $\S \S 3$ ff. Qualifikation des amtlichen Leichenschauers (z. B. Rechtsmediziner), Datenschutz, Dokumentation, Verbotsvorschriften, Straf- und Bußgeldverfahren, Schlussvorschriften usw. 
Hirntod mit dem menschlichen Tod gleichgesetzt oder abgelehnt ${ }^{162}$. Der Philosoph Hans Jonas beschreibt den Moment des Hirntodes als Zeitpunkt in dem das »Lebendsein im Zwielicht des Zweifelhaften läge«, während der Arzt Johann Friedrich Spittler in diesem Moment »im hirntoten, noch überlebenden übrigen Körper Lebendigkeit nur noch in einem biologischen, nicht mehr in einem anthropologischen (humanen) Sinn findet « und der Jurist Reinhold Zippelius zu Fragen in den Randgebieten des Lebensrechts ausführt »Wo immer aber hier der Jurist die Entscheidung an sich zieht, sollte er sich der Not des Handelnden und der Unzulänglichkeit der juristischen Maßstäbe bewusst sein ${ }^{163}$.

Ungeachtet aller Zweifel und definitorischen Schwierigkeiten sind diese existentiellen Fragen zum Ende des menschlichen Individuums so wesentlich, dass es einer Konkretisierung des Todeszeitpunktes oder der Todeszeitpunkte und deren Feststellung durch den demokratisch legitimierten Gesetzgeber bedarf, wann der Mensch jenseits der Mindestvoraussetzung als Organspender in Frage zu kommen, als tot anzusehen ist, um den letzten Augenblick des menschlichen Individuums auch mit verschiedenen Todesdefinitionen und Todesfeststellungszeitpunkten rechtlich klar zu bestimmen. Gerade besonders grundrechtsintensive Eingriffe insbesondere im Bereich des Lebens bzw. seinem Ende müssen nach der Wesentlichkeitslehre in einem Parlamentsgesetz eindeutig und wesentlich geregelt werden. Da das Grundrechtsgut Leben »schlechthin die allgemeine und elementarste Voraussetzung für Grundrechte überhaupt und damit nicht zuletzt auch die vitale Basis der Menschenwürde« darstellt, ist eine Delegation gesetzgeberischer Aufgaben in diesem grundrechtsrelevanten Bereich auf andere, wenn auch fachkompetente, aber nicht demokratisch legitimierte Organisationen kaum begründbar. In einem anerkannten Prozess des Sterbens mit einem mehr oder minder langen Verlauf stellen der Zeitpunkt oder die Zeitpunkte des Todes Gegenstände normativer, kultureller und gesellschaftspolitischer Festlegungen und Vereinbarungen unter Mithilfe vom Gesetzgeber vorzugebender medizinischer Kriterien dar, die dem Einzelnen einen nachvollziehbaren und verständlichen Rahmen vorgeben, um z. B. im Rahmen einer Patientenverfügung zur Achtung seines Selbstbestimmungsrechts bei Vorliegen des Gesamthirntods als Organspender zur Verfügung zu stehen oder durch sofortiges Abstellen der intensivmedizinischen Apparate den Stillstand von Atmung, Herz- und Kreislauf herbeigeführt zu bekommen.

Letztendlich ist eine solche prämortale selbstbestimmte und freie Entscheidung des Patienten, die zu beachten ist, irreversibel bindend. Das individuelle Sterben und der individuelle Tod des Patienten kann dann vielleicht, wie von Goethes Prometheus seiner Tochter Pandora beschrieben, eintreten: »Da ist ein Augenblick, der alles erfüllt. Alles, was wir gesehnt, geträumt, gehofft, gefürchtet, meine Beste, - das ist der Tod!... Wenn aus dem innerst tiefsten Grunde, du ganz erschüttert alles fühlst, was Freud und

161 Kliegel, Ist der Mensch sein Gehirn? Ethik Med 2000, S. 75 (77).

162 Quante, Personales Leben und menschlicher Tod. Personale Identität als Prinzip der biomedizinischen Ethik, 2002, S. 126 a. A. Rehbock, Rezension zu Quante, Ethik in der Medizin, 2003, 135 (136).

163 Jonas in: Hoff/in der Schmitten (Hrsg.) Wann ist der Mensch tot? Organverpflanzung und Hirntodkriterium, 1994, S. 24; Spittler, a. a. O. S. 118; Zippelius, a. a. O. 659 (663). 
Schmerzen jemals dir ergossen, im Sturm dein Herz erschwillt, in Tränen sich erleichtern will und seine Glut vermehrt, und alles klingt an dir und bebt und zittert, und all die Sinne dir vergehn, und du dir zu vergehen scheinst und sinkst, und alles um dich her, versinkt in Nacht, und du, in inner eigenem Gefühle, umfassest eine Welt: Dann stirbt der Mensch. ${ }^{164}$.

164 Goethe, Prometheus, ca. 1773, Dramatisches Fragment, Erster Akt. 